\title{
Groundwater Maps of the Hanford Site, December 1994
}

\author{
J. A. Serkowski \\ M. J. Hartman \\ M. D. Sweeney
}

Date Published

June 1995

\section{DISCLAIMER}

This report was prepared as an account of work sponsored by an agency of the United States Government. Neither the United States Government nor any agency thereof, nor any of their employees, makes any warranty, express or implied, or assumes any legal liability or responsibility for the accuracy, completeness, or usefulness of any information, apparatus, product, or process disclosed, or represents that its use would not infringe privately owned rights. Reference herein to any specific commercial product, process, or service by trade name, trademark, manufacturer, or otherwise does not necessarily constitute or imply its endorsement, recommendation, or favoring by the United States Government or any agency thereof. The views and opinions of authors expressed herein do not necessarily state or reflect those of the United States Government or any agency thereof.

Prepared for the U.S. Department of Energy Office of Environmental Restoration and Waste Management

\section{Q. Westinghouse P.O Box 1970 Hanford Company Richland, Washington}

Hanford Operations and Engineering Contractor for the U.S. Department of Energy under Contract DE-AC06-87RL10930 


\section{DISCLAIMER}

Portions of this document may be illegible in electronic image products. Images are produced from the best available original document. 


\section{RELEASE AUTHORIZATION}

Document Number: WHC-EP-0394-10

Document Title: Groundwater Maps of the Hanford Site, December 1994

Release Date: August 21, 1995

This document was reviewed following the procedures described in WHC-CM-3-4 and is:

APPROVED FOR PUBLIC RELEASE

WHC Information Release Administration Specialist:

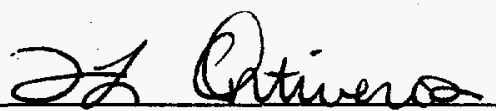

August 21, 1995

T.L. Ontiveros 
WHC-EP-0394-10

CONTENTS

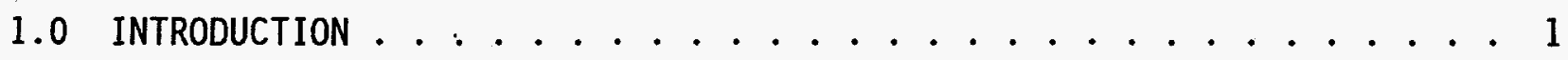

2.0 DATA COLLECTION, MANAGEMENT, EVALUATION, AND PRESENTATION $\ldots \ldots . .1$

3.0 HANFORD SITE MAPS ................... 2

3.1100 AREAS MAPS . . . . . . . . . . . . . . . . . 3

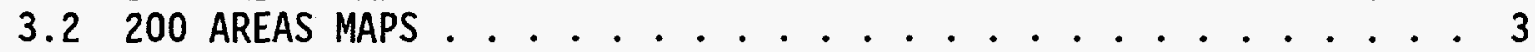

$3.3300 / 1100$ AREAS MAPS $\ldots \ldots \ldots . \ldots . \ldots . \ldots 4$

4.0 REFERENCES ......................... 4

APPENDIX

A Water Level Measurement Data ................ A-1

\section{LIST OF FIGURES}

1. Hanford Site Location Map . . . . . . . . . . . . . 7

2. Hanford Site Water Table ................ 9

3. 100 Area Location Map ................. 11

4. 100 Area Water Table ................ . 13

5. 200 Area Location Map . . . . . . . . . . . . . . . . 15

6. 200 Area Water Table ............... . . 17

7. 300 Area Location Map ......... . . . . . . . . 19

8. 300 Area Water Table .................. 21 
WHC-EP-0394-10

This page intentionally left blank. 


\section{WHC-EP-0394-10 \\ GROUNDWATER MAPS OF THE HANFORD SITE DECEMBER 1994}

\subsection{INTRODUCTION}

The Groundwater Maps of the Hanford Site. December 1994 is a continuation of a series of reports (see Serkowski et al 1994) that document the configuration of the uppermost unconfined aquifer beneath the Hanford Site (Figure 1). This series presents the results of the semiannual water level measurement program and the water table maps generated from these measurements. The reports document the changes in the groundwater level at the Hanford Site during the transition from nuclear material production to environmental restoration and remediation. In addition, these reports provide water level data to support the various site characterization and groundwater monitoring programs currently in progress on the Hanford Site.

Groundwater Maps of the Hanford Site is prepared for the U.S. Department of Energy, by the Hanford Site Operations and Engineering Contractor, Westinghouse Hanford Company (WHC). This document fulfills reporting requirements specified in WHC-CM-7-5, Section 8.0 "Water Quality" (WHC 1993a) and described in the environmental monitoring plan for the Hanford Site. (DOERL 1991)

This document highlights the three major operations areas (the 100, 200 and $300 / 1100$ Areas) where wastes were discharged to the soil. Each area includes a summary discussion of the data, a well index map, and a contoured map of the water table surface. Appendix A contains all of the data collected for this program.

The National Geodetic Vertical Datum of 1988 (NGVD88) is used as the vertical datum and Washington State Lambert coordinates are used for horizontal location of the wells. Elevation data were converted from feet to meters for this report.

\subsection{DATA COLLECTION, MANAGEMENT, EVALUATION, AND PRESENTATION}

During December 1994, the depth to groundwater was measured in 814 Hanford Site wells by personnel from the Well Services Group under the direction of the Groundwater Management Group project leader. Wells were selected for inclusion in this report based on the following criteria:

- The well must monitor only the uppermost $(<15.5 \mathrm{~m}[50 \mathrm{ft}])$ part of the aquifer

- The screened interval of the we11 should not exceed $15 \mathrm{~m}$ and the water table should intersect the screened interval. Exceptions 
WHC-EP-0394-10

were made where no alternate wells exist and vertical gradients were not considered significant.

- Well location and elevation must be accurately known.

The procedure for measuring the depth to water and recording the data is contained in EII 10.2, Environmental Investigation and Site Characterization Manual WHC-CM-7-7 (WHC 1993b). Field data were collected using the - Groundwater Monitoring System, which employs a barcode interface to enter readings that have been taken manually with a steel tape from the reference point at the top of the well casing. The barcode hand-held computer downloaded the field data to a supervisory workstation which stores all groundwater level measurements. These data were transferred to the Hanford Enyironmental Information System (HEIS) data base and were later downloaded to a Paradox ${ }^{\mathrm{tm}}$ application program called GeoDAT to help organize, evaluate, and tabulate the data.

of the 814 wells measured in June 1994,442 were selected to create a water table surface model. A model grid of the remaining wells was generated using a minimum tension algorithm available in 2 Earthvision ${ }^{\text {tm }}$ geologic modeling program. The modeling software then created contour 1 ines of equal water table elevations from the model grid. The contours and annotation information were modified on the ${ }^{3}$ ARC $/$ INFO ${ }^{\text {tm }}$ geographic information system software. Contours are not present in areas where the basalt surface is believed to be above the water table, based on Connelly (1992a and 1992b).

Hydrologists familiar with regional and local groundwater properties reviewed the maps to evaluate data interpolations made by the computer model. The contours were adjusted manually to reflect the known hydrologic environment.

\subsection{HANFORD SITE MAPS}

This section summarizes the results of the water table surface model generated from the December 1994 data. Figure 1 shows the relative locations of the detail facility area maps included in this document. To provide

\footnotetext{
${ }^{1}$ Paradox ${ }^{\text {tm }}$ - Borland International, 1800 Green Hills Road P.0. Box 660001, Scotts Valley, CA 95067-0001

${ }^{2} A R C / I N F 0^{\text {tm }}$ - Environmental Systems Research Institute, Inc., Redlands, CA 92373-8100

${ }^{3}$ Earthvision ${ }^{\text {tm }}$ - Dynamic Graphics, Inc., 1015 Atlantic Avenue Alameda, CA 94501
} 


$$
\text { WHC-EP-0394-10 }
$$

context, Figure 2 shows the water table surface for the entire Hanford Site. The detail maps are enlargements of this same site-wide model.

\subsection{AREAS MAPS}

For the purposes of this report, the 100 Areas comprise the various 100 Area reactor facilities and the surrounding land south of the Columbia River and north of Gable Mountain and Gable Butte. Reactor operations have ceased in all of the facilities, and environmental restoration activities are in progress. Maps for this area include: Figure 3, Index Map of the 100 Areas Groundwater Monitoring Wells; and Figure 4, 100 Areas Water Table. Some of the wells used have screened intervals exceeding 15 meters within the top of the unconfined aquifer since the vertical gradients are not believed to be significant in this area.

Throughout most of the map area, groundwater flows from the unconfined aquifer into the Columbia River. West of the $100-B / C$ Area, water is believed to flow from the river into the aquifer.

The high water levels north of Gable Mountain are consistent with past measurements. Driller's logs indicate the presence of fine-grained sediments in this area. The high water levels may be remnants of artificial recharge from pre-Hanford irrigation, or may represent perched groundwater. The steep groundwater gradient in the southeastern portion of the map area is believed to result from the influence of the fine-grained sediments.

\subsection{AREAS MAPS}

These maps encompass the 200 East and 200 West Areas and the surrounding vicinity on the Hanford Site that was once referred to as the Separations Area. There are several active and many inactive liquid waste disposal facilities in this region. The 200 Areas set of maps consists of: Figure 5, 200 Areas Monitoring Well Index Map; and, Figure 6, 200 Areas Water Tab7e.

Three facilities are notable in their impact on the water levels in this area: $U$ Pond $(216-U-10)$, located in the southwestern corner of 200 West Area, $Z$ Plant (234-5), north of the U Pond Site, and B Pond (216-B-3), located east of 200 East Area. U Pond was deactivated in 1984 after 40 years of use as a disposal site for large volumes of liquid wastes. The relatively low hydraulic conductivity found beneath 200 West Area coupled with continued effluent discharge in cribs associated with $Z$ Plant has helped to maintain a significant water table mound under 200 West Area. Since 1984, the high point of the groundwater mound has shifted northward from $U$ pond, toward $Z$ PI ant. A steep gradient occurs east of 200 West Area as the water table intersects the higher conductivity sediments beneath 200 East Area. With higher conductivities, the water table beneath the 200 East Area is generally flat. $B$ Pond, which received significant volumes of 1 iquid effluent, has a residual 
WHC-EP-0394-10

groundwater mound that influences groundwater travel direction over a wide area.

\section{$3.3 \quad 300 / 1100$ AREAS MAPS}

This section contains a discussion on the December 1994 water table measurements for the 300 Area and the adjacent 1100 Area. Liquid waste disposal continues only at the 300 Area Process Trenches, though clean river water is discharged during the summer months at the Richland well field recharge ponds located east of the 1100 Area. The 300/1100 Area set of maps consists of: Figure 7, 300 Area Monitoring Well Index Map; and, Figure 8, 300 Areas Water Table.

The unconfined aquifer within this area is contained in the Ringold Formation and the Hanford formation (Swanson et al, 1992). The top of the unconfined aquifer is close to the Hanford/Ringold contact. Generally, west of an imaginary north-south line near the west boundary of the 300 Area the unconfined aquifer is within the Ringold Formation. East of the line it is within the Hanford formation, which is more permeable than the Ringold and may be expected to permit higher flow velocities.

The major influences on water table elevations in the map area are river fluctuations, irrigation, and river water recharged into the City of Richland well field near the 1100 Area. Water table elevation may also be dependent on recharge from the Yakima River to the west. Combined with high permeabilities in the Hanford formation, these influences result in a complex flow regime where local flow directions are difficult to predict.

\subsection{REFERENCES}

Connelly, M. P., B. H. Ford, and J. V. Borghese, 1992a, Hydrogeologic Model for the 200 West Groundwater Aggregate Area, WHC-SD-EN-TI-014, Westinghouse Hanford Company, Richland, Washington.

Connelly, M. P., B. H. Ford, J. W. Lindberg, S. J. Trent, C. D. Delaney, and J. V. Borghese, 1992b, Hydrogeologic Model for the 200 East Groundwater Aggregate Area, WHC-SD-EN-TI-019, West inghouse Hanford Company, Richland, Washington

DOE-RL, 1991, Environmental Monitoring P7an, DOE/RL 91-50, U.S. Department of Energy, Richland Field Office, Richland, Washington.

Serkowski, J. A., W. A. Jordan, and M. J. Hartman, 1994, Ground Water Maps of the Hanford Site--June 1994, WHC-EP-0394-9, Westinghouse Hanford Company, Richland, Washington. 
Swanson, L. C., G. G. Kelty, K. A. Lindsey, K. R. Simpson, R. K. Price, and S. D. Consort, Phase I Hydrogeologic Summary of the 300-FF-5 Operable Unit, 300 Area, WHC-SD-EN-TI-052, Westinghouse Hanford Company, Richland, Washington.

Westinghouse Hanford Company, 1993a, Environmental Compliance, WHC-CM-7-5, Westinghouse Hanford Company, Richland, Washington.

Westinghouse Hanford Company, 1993b, Environmental Investigations and Site Characterization Manual, WHC-CM-7-7, Westinghouse Hanford Company, Richland, Washington. 
WHC-EP-0394-10

This page intentionally left blank. 


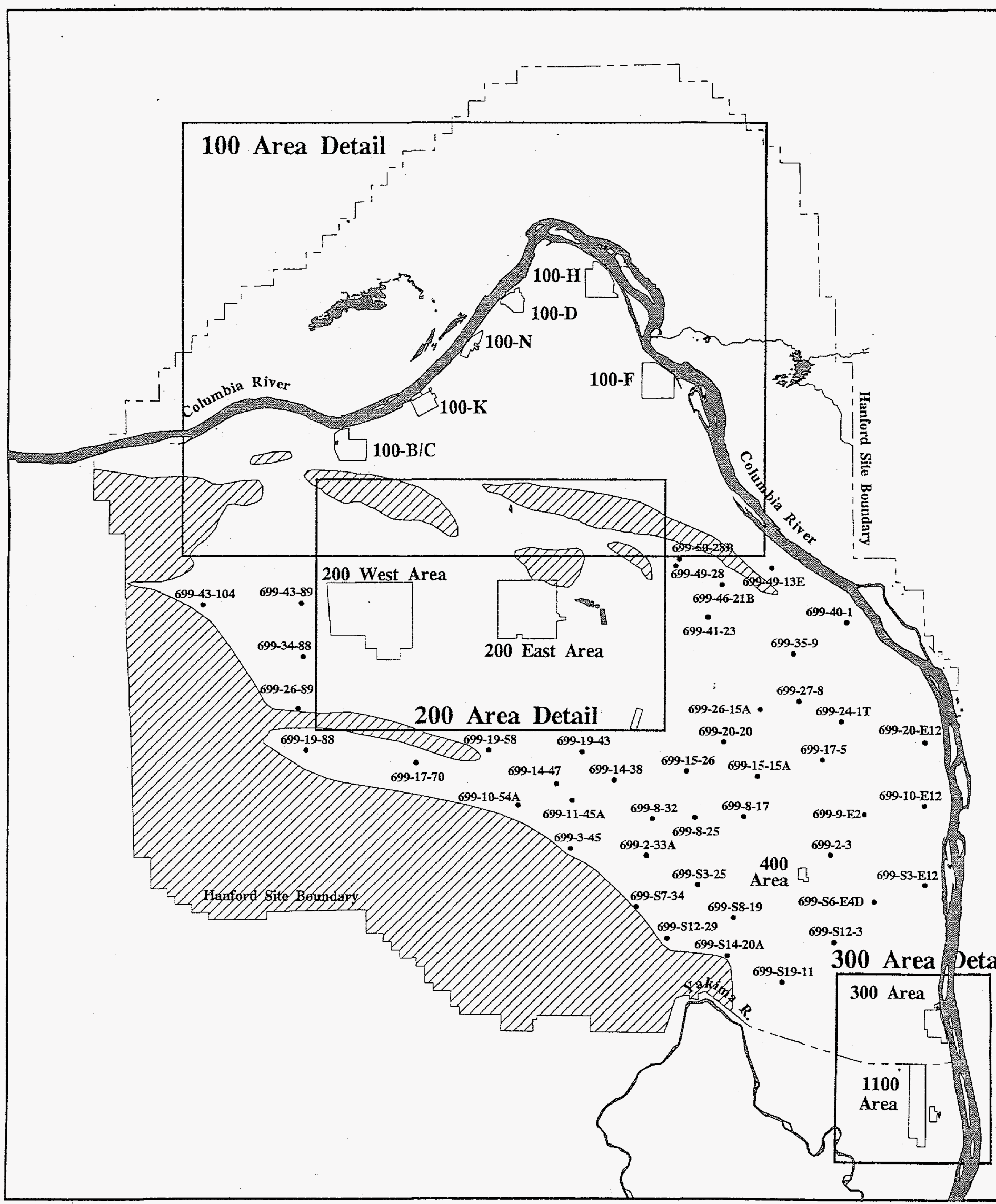




\section{Hanford Site} Index Map Dec 1994

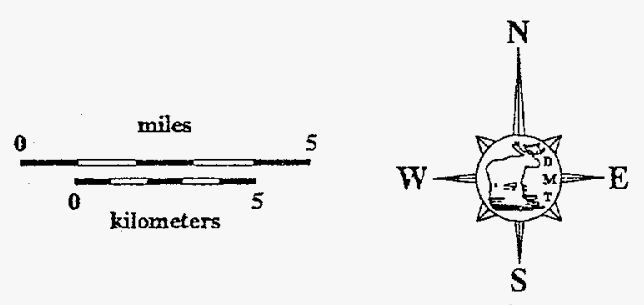

- Well location used for creating this model

Ponds, lakes, and rivers

Areas where basalt surface is generally above the water table

Prepared by the Data Management Team, Hanford Technical Services, Westinghouse Hanford Company.

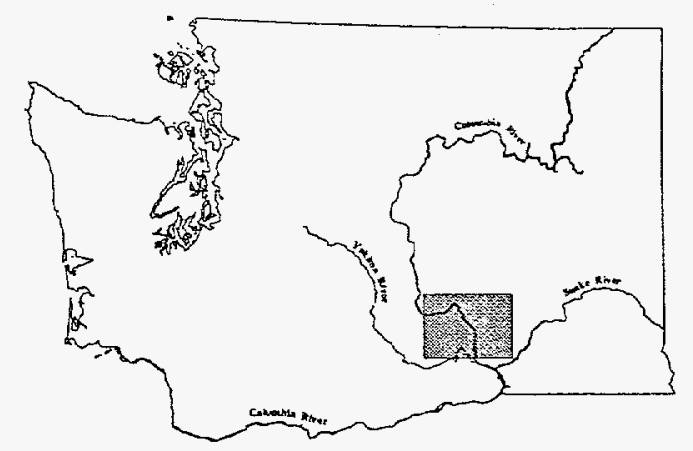




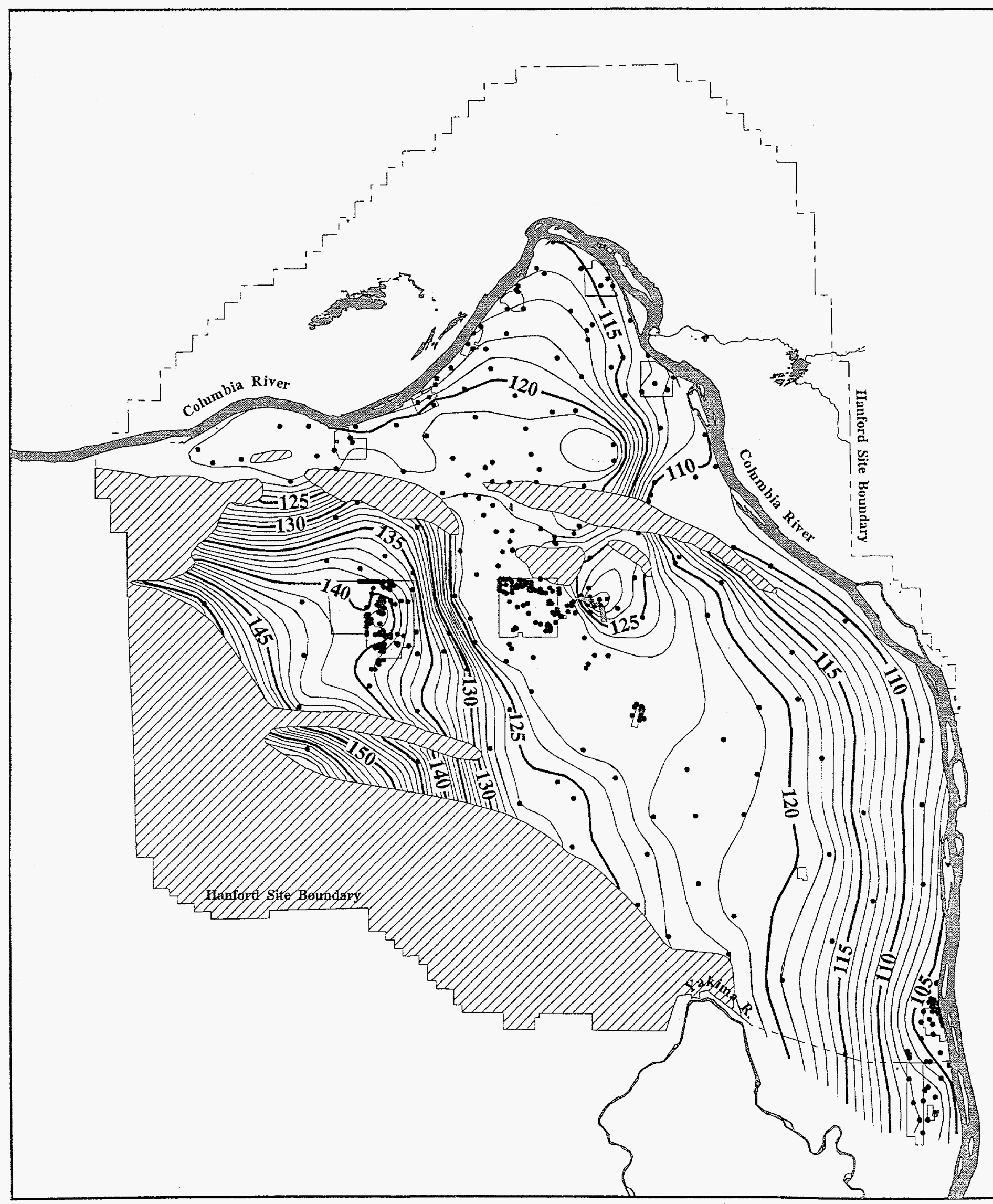




\section{Hanford Site Water Table Dec 1994 \\ 0
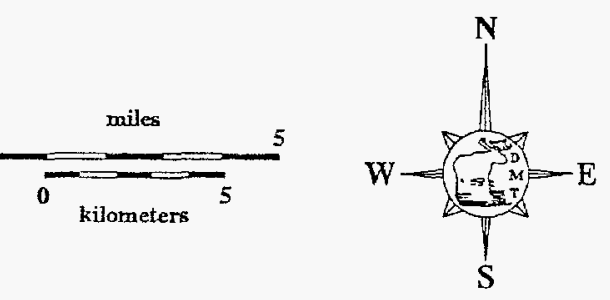

Well location used for creating

- this model $-122-\begin{aligned} & \text { Water level contour } \\ & (\mathrm{m} \text { above } \mathrm{msl})\end{aligned}$

Ponds, lakes, and rivers

Areas where basalt surface is generally above the water table

Prepared by the Data Management Team, Hanford Technical Services, Westinghouse Hanford Company.

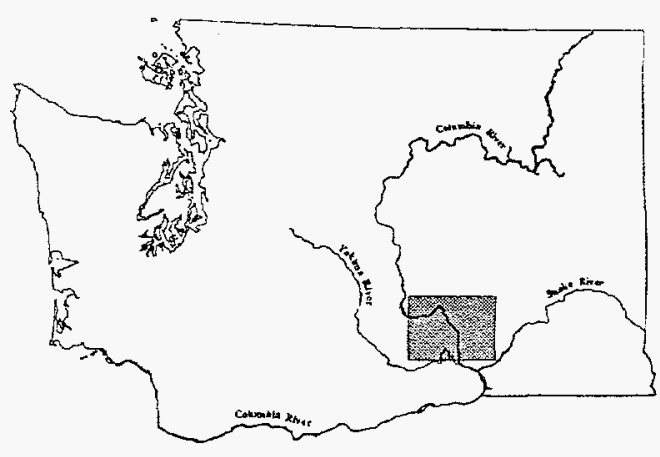

wl hs.map-062895

Figure 2. Hanford Site Water Table Map. 


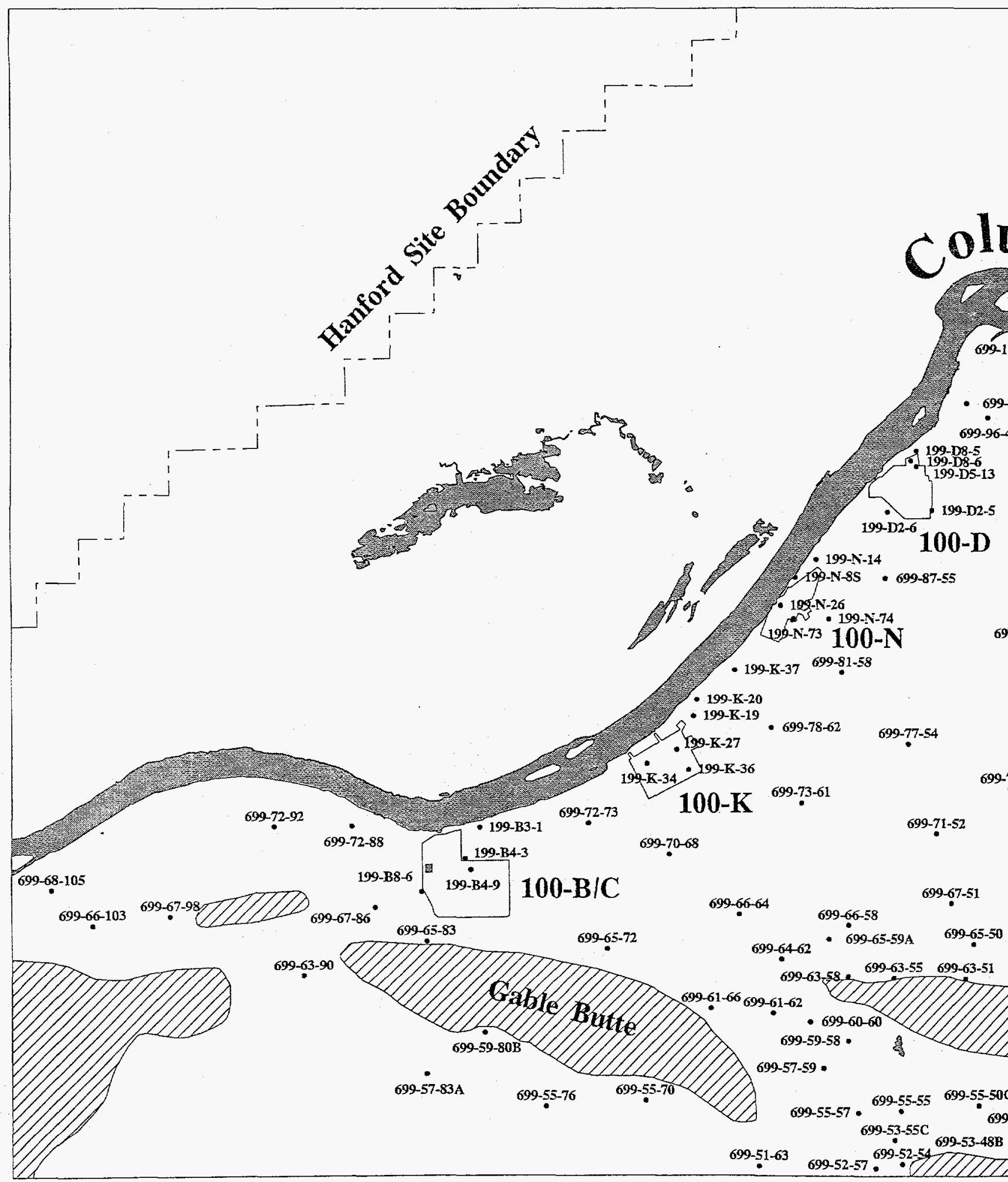




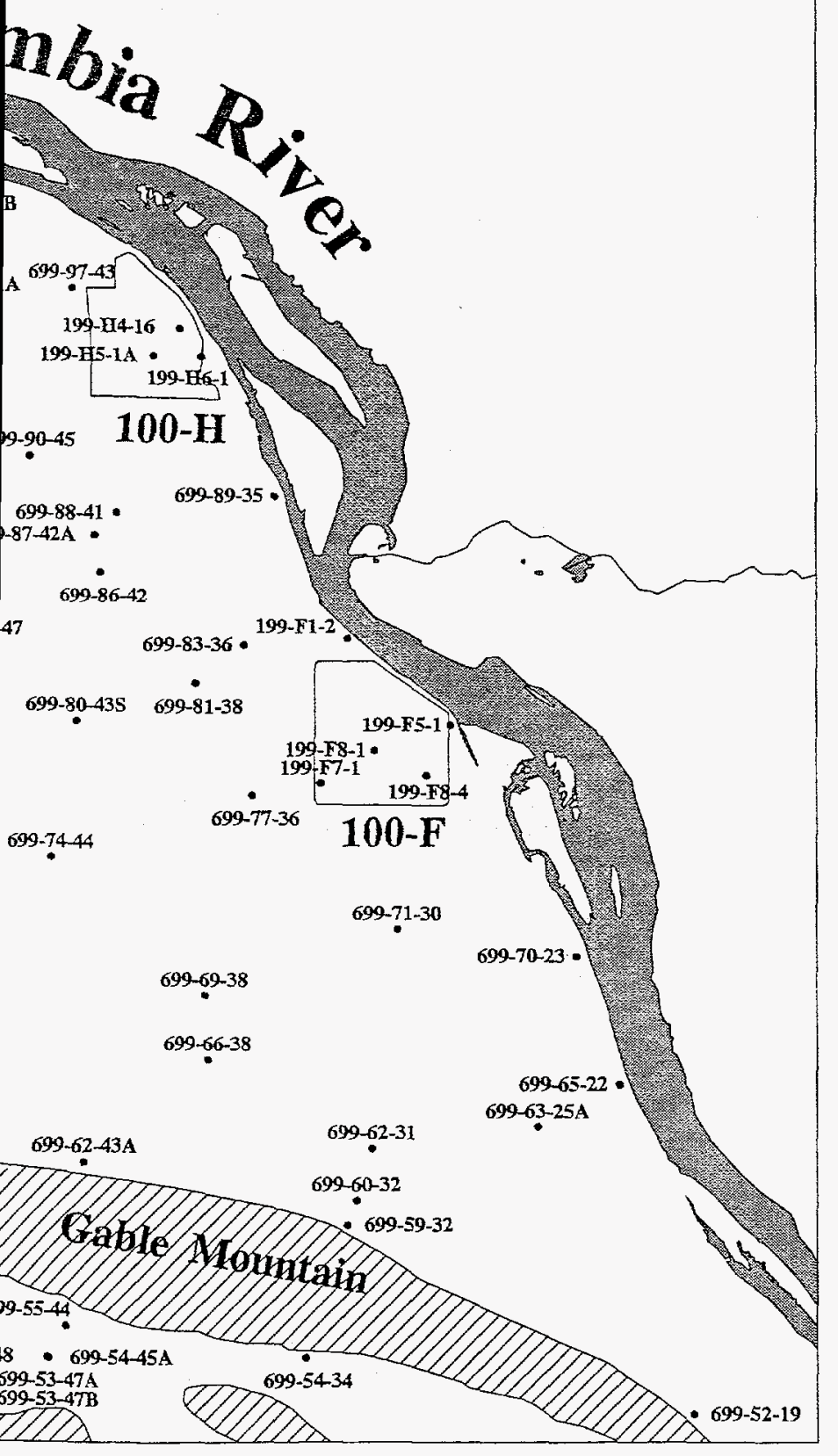

\section{Areas Index Map Dec 1994

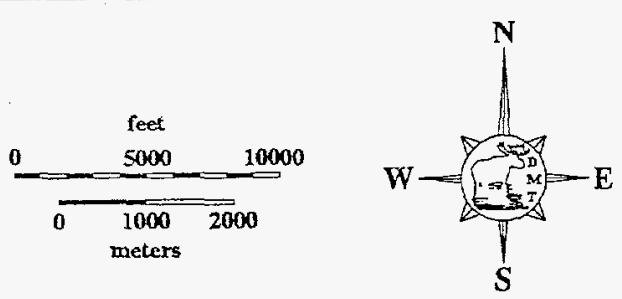

Prepared by the Data Management Team, Hanford Technical Services, Westinghouse Hanford Company.

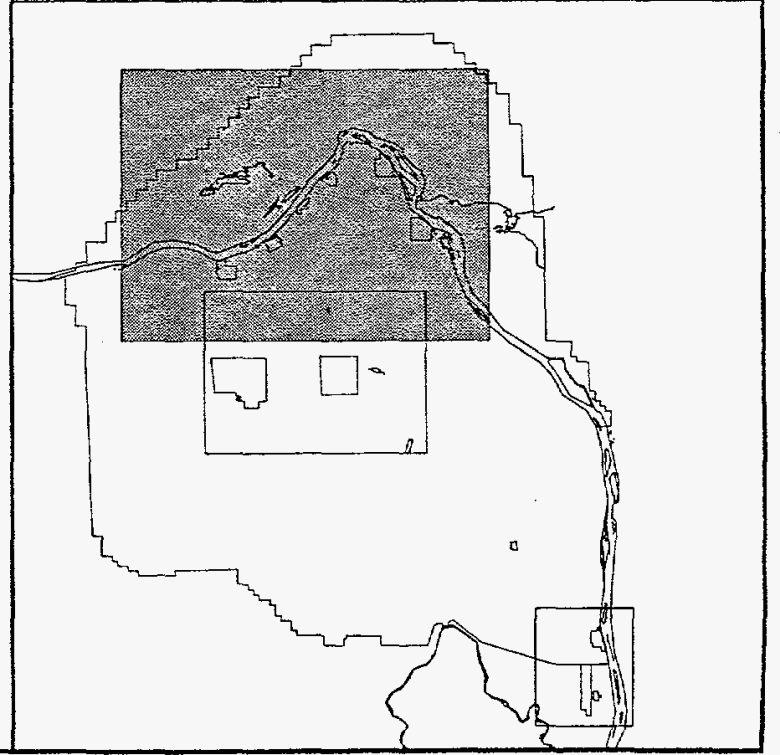

Figure 3. 100 Area Location Map. 


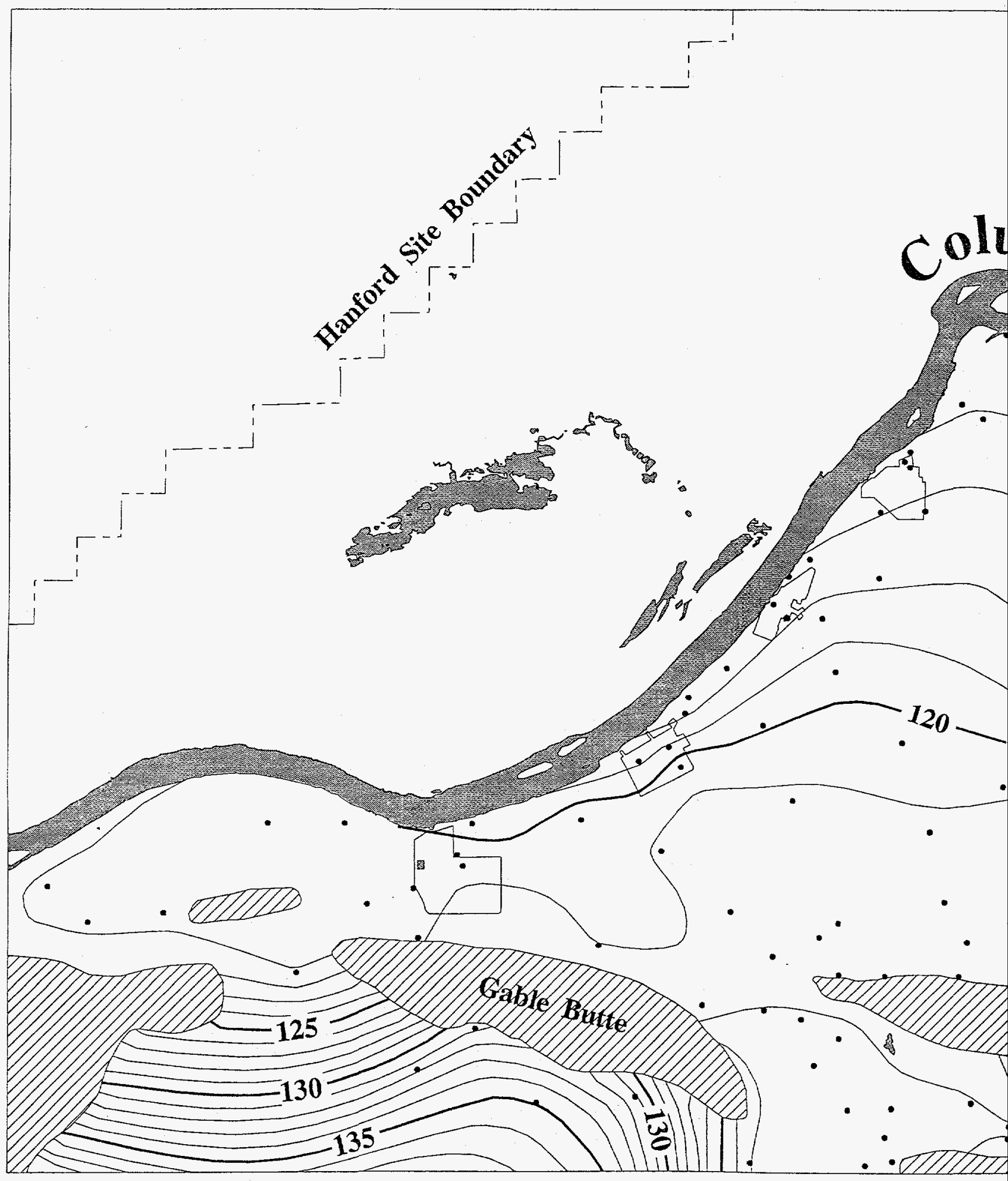



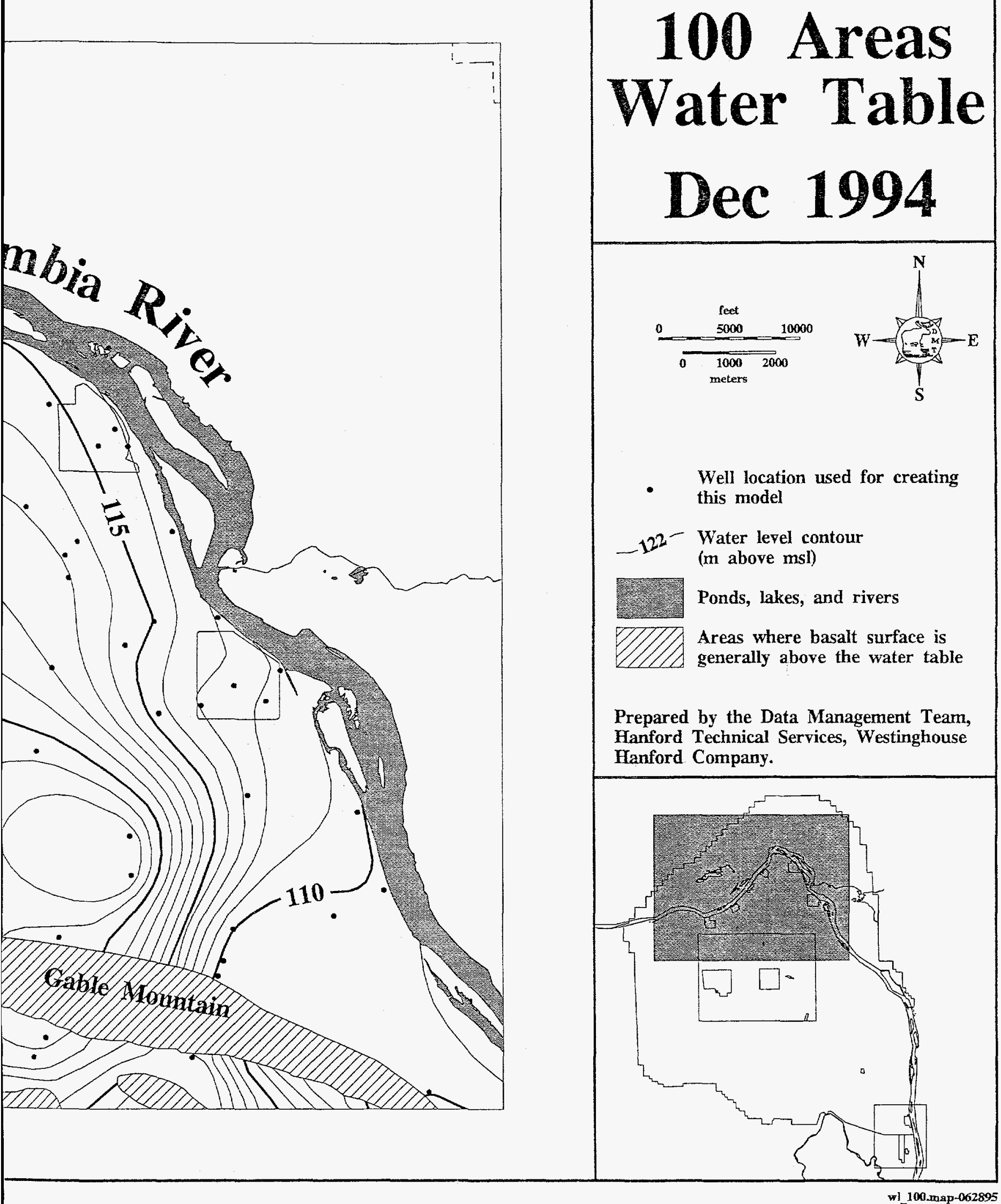

Figure 4. 100 Area Water Table. 


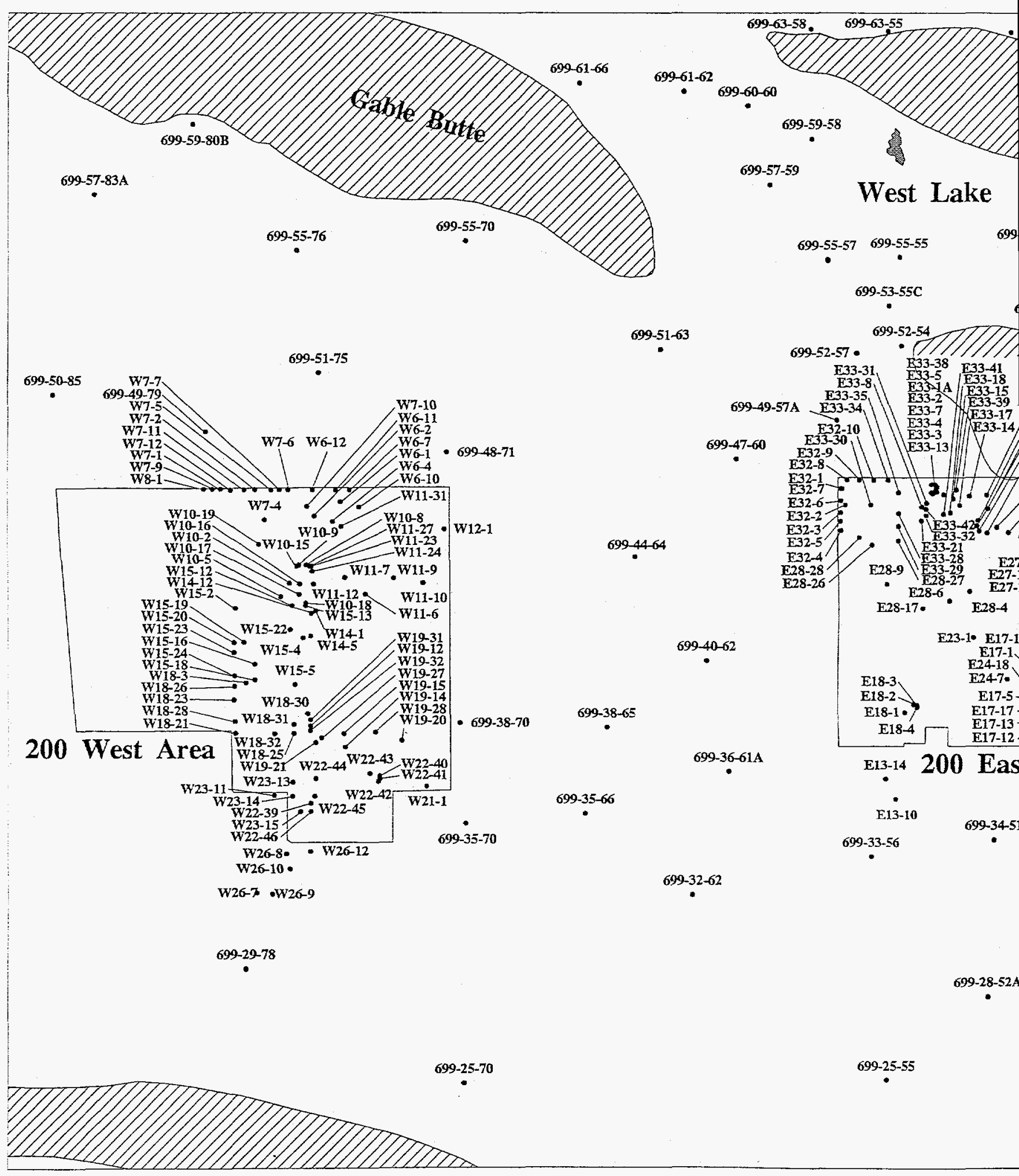




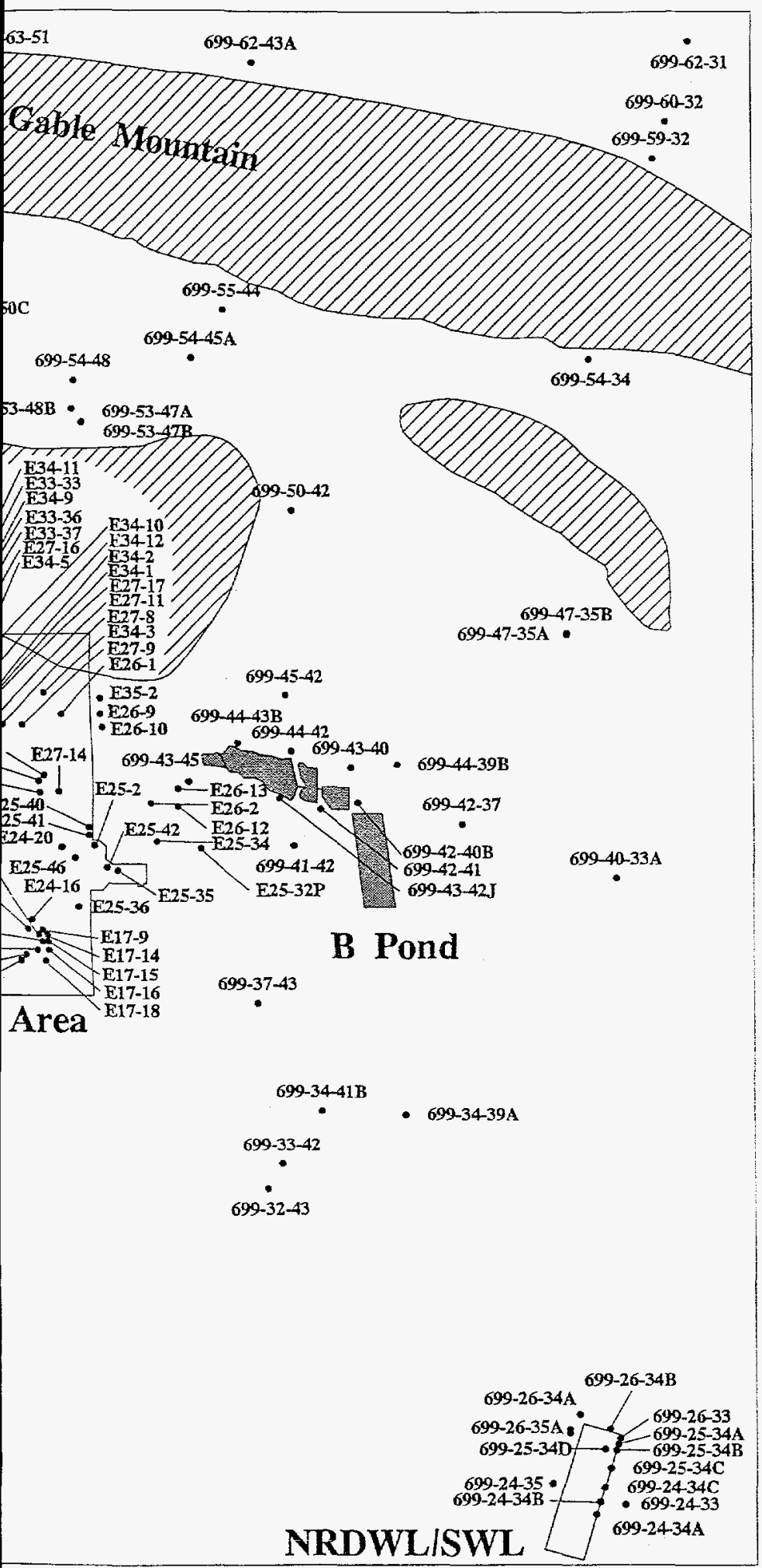

200 Areas Index Map Dec 1994

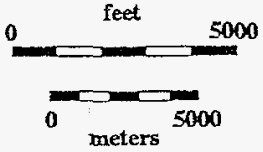

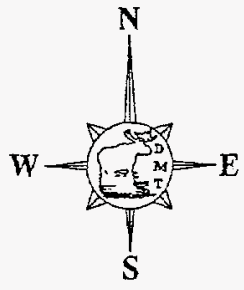

- Well location used for creating this model

Ponds, lakes, and rivers

777 Areas where basalt surface is generally above the water table

Prepared by the Data Management Team, Hanford Technical Services, Westinghouse Hanford Company.

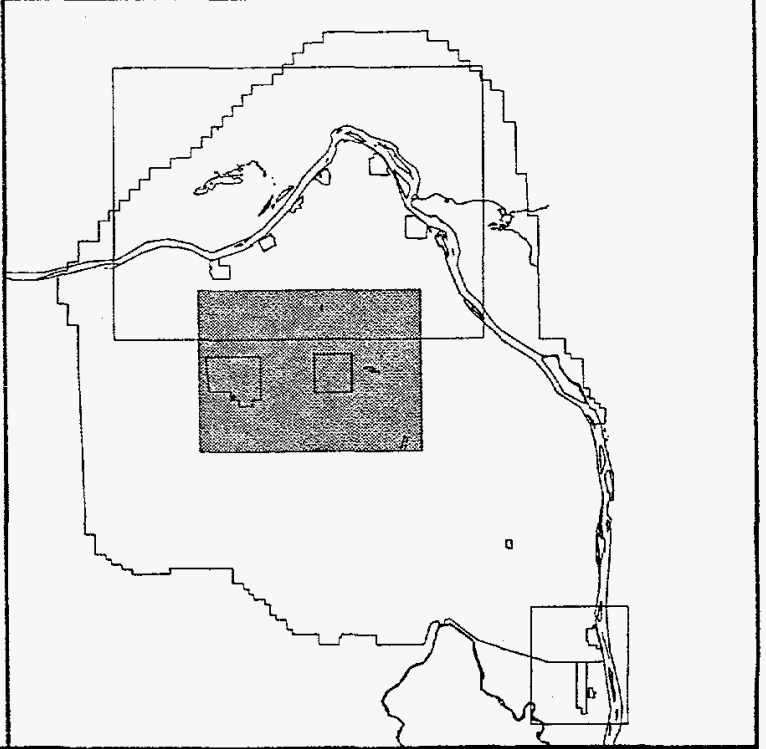

index 200.map-070595

Figure 5. 200 Area Location Map. 


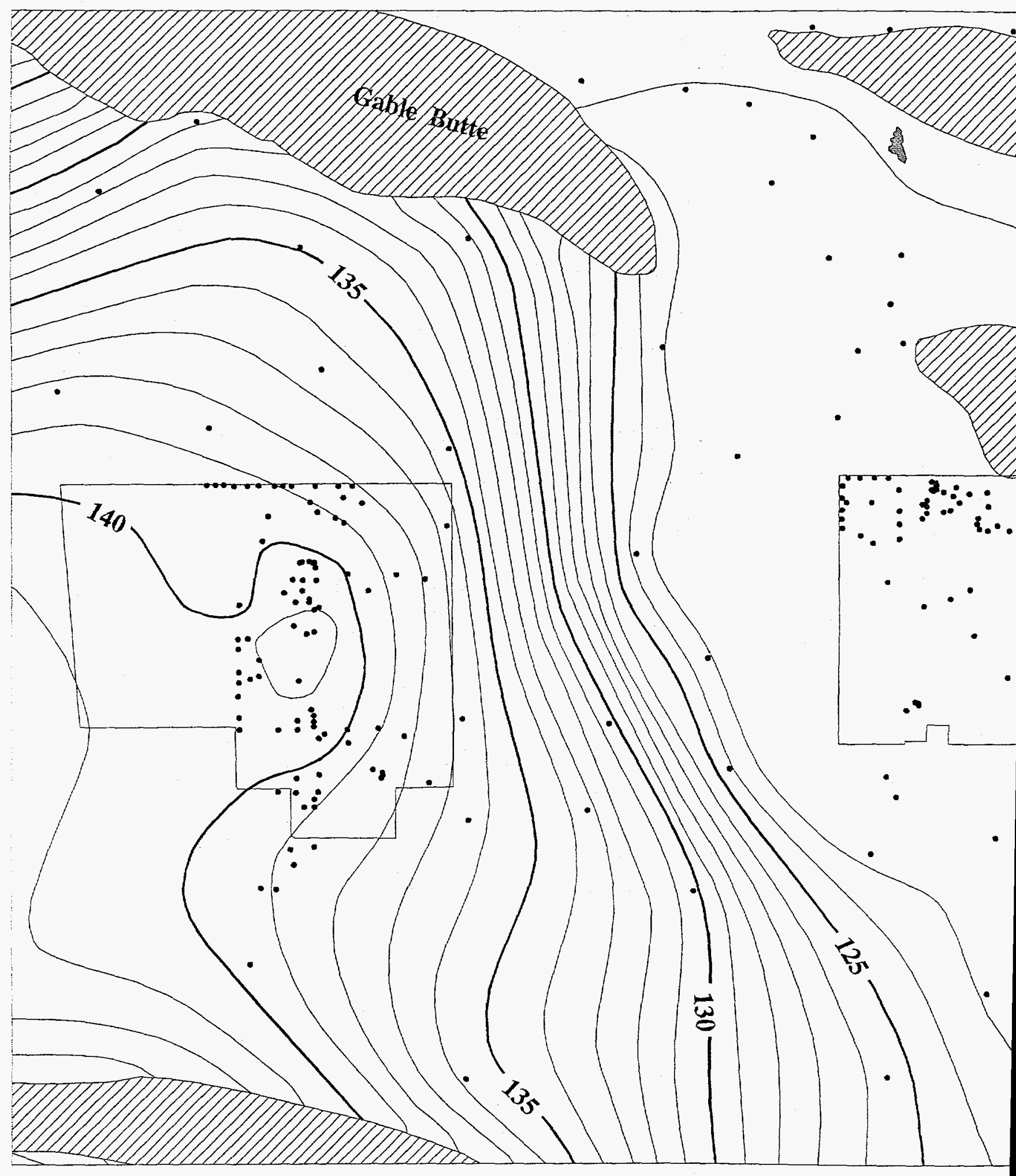




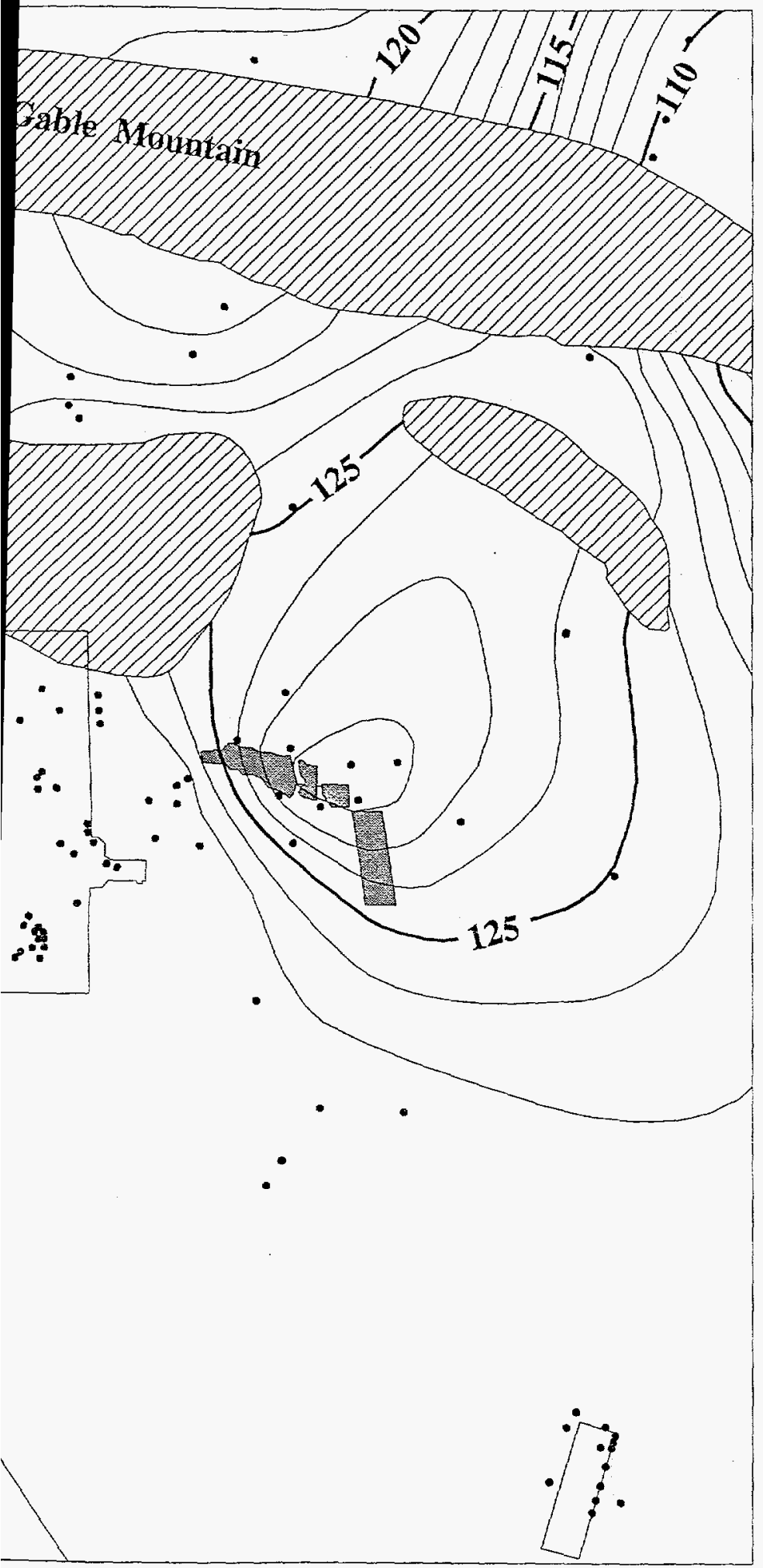

\section{Areas} Water Table Dec 1994
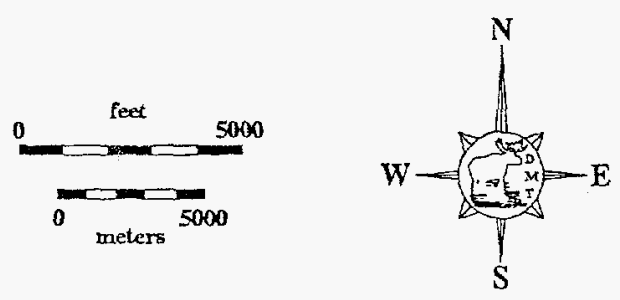

- Well location used for creating this model

-122 - Water level contour ( $\mathrm{m}$ above $\mathrm{msl}$ )

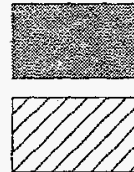
Ponds, lakes, and rivers

Areas where basalt surface is generally above the water table

Prepared by the Data Management Team, Hanford Technical Services, Westinghouse Hanford Company.

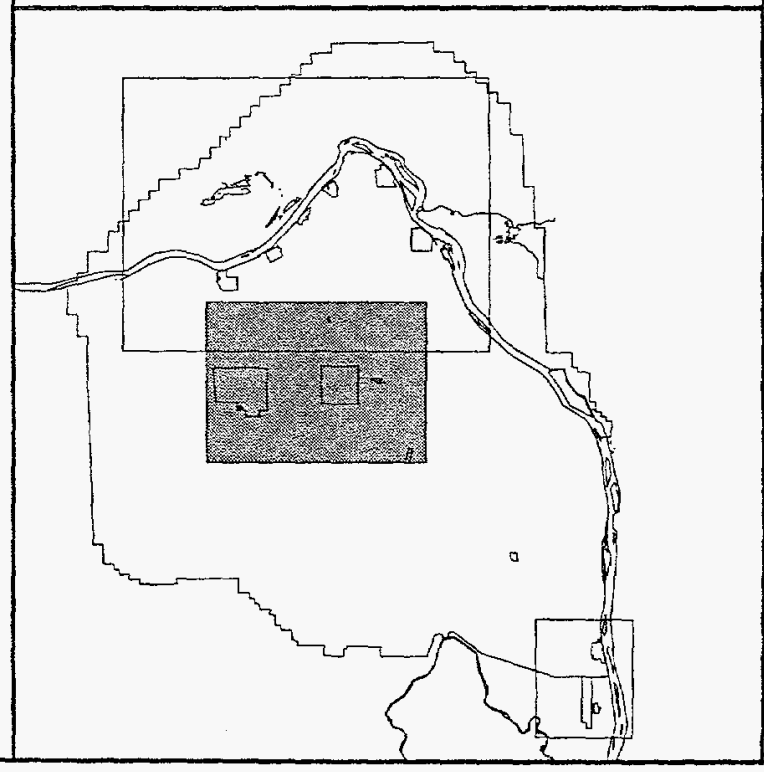

w1_200.map-162895

Figure 6. 200 Area Water Table. 


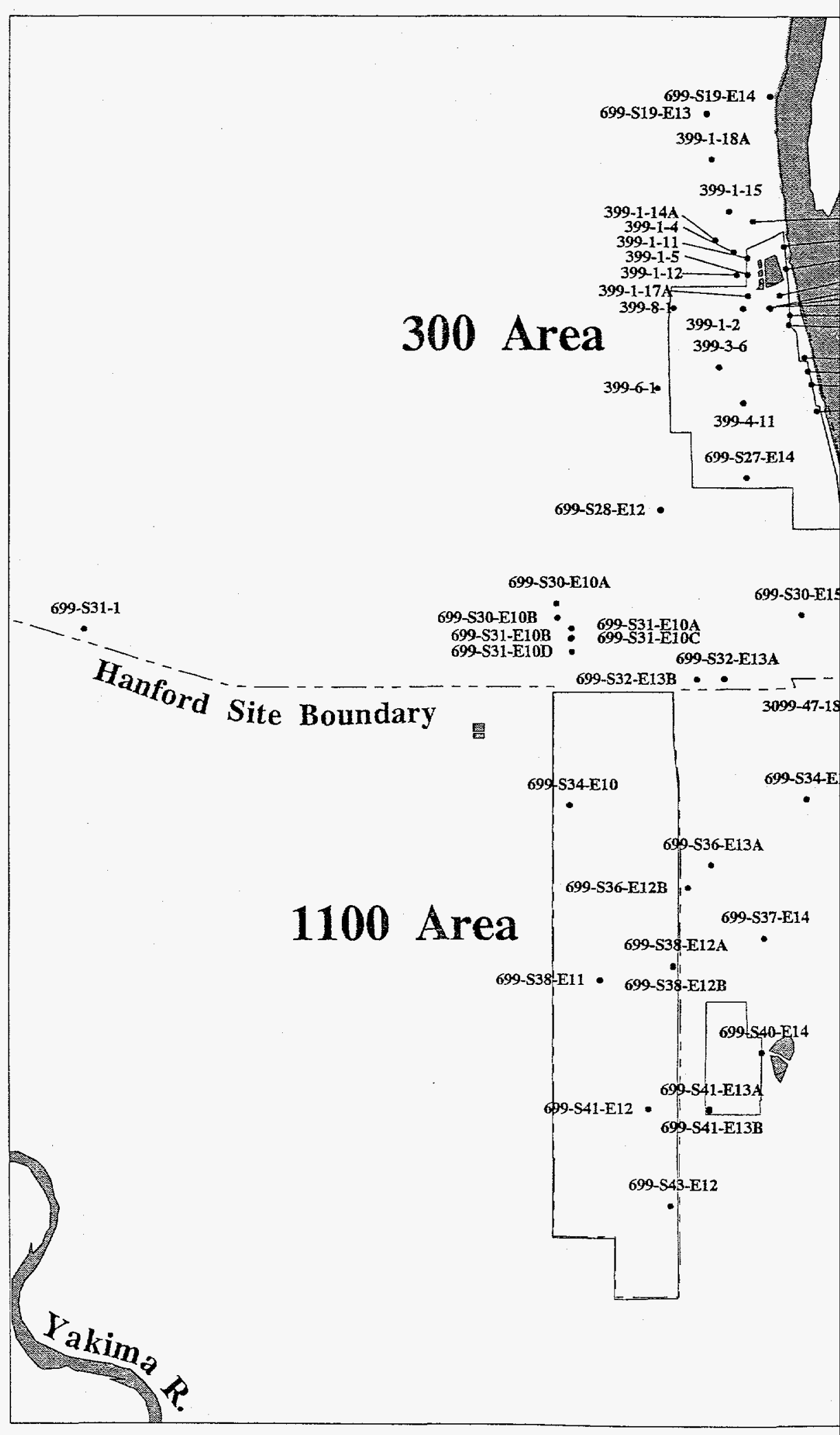




$$
2 .
$$




\section{Area}

Water Table

Dec 1994
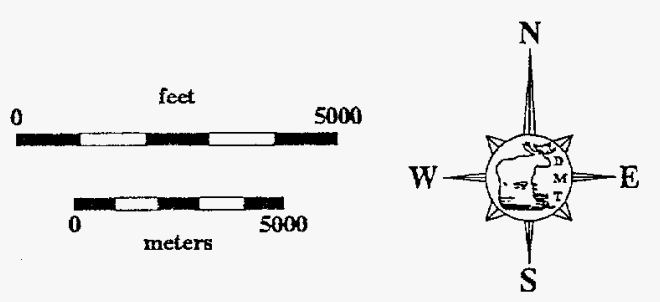

Well location used for creating

- this model

- Water level contour
(m above msl)

Ponds, lakes, and rivers

$D D$ Areas where basalt surface is generally above the water table

Prepared by the Data Management Team, Hanford Technical Services, Westinghouse Hanford Company.

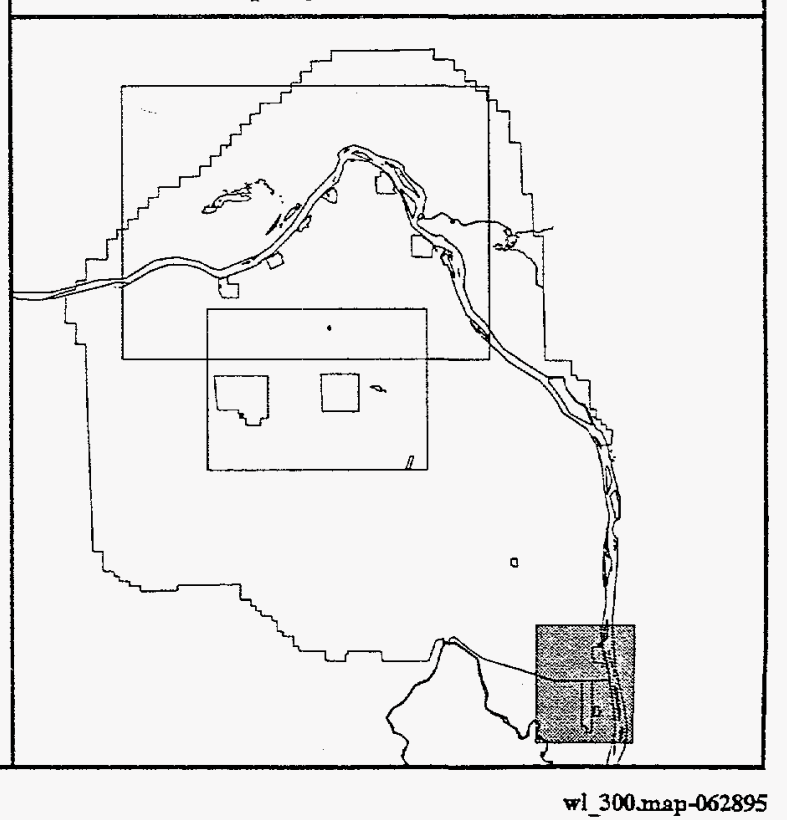

Figure 8. 300 Area Water Table. 
WHC-EP-0394-10

APPENDIX A

WATER LEVEL MEASUREMENT DATA

A-1 
WHC-EP-0394-10

This page intentionally left blank. 
WHC-EP-0394-10

Appendix A: December 1994 Water Level Measurement Data (Sheet 1 of 13)

\begin{tabular}{|c|c|c|c|}
\hline We11 & $\begin{array}{l}\text { Depth to } \\
\text { Water (m) }\end{array}$ & $\begin{array}{r}\text { Elevation, } \\
\text { Adjusted Casing }\end{array}$ & $\begin{array}{l}\text { above msl } \\
\text { Water Leve1 }\end{array}$ \\
\hline $\begin{array}{l}199-B 3-1 \\
199-B 4-3 \\
199-B 4-9 \\
199-B 8-6 \\
199-D 2-5\end{array}$ & $\begin{array}{l}14.40 \\
20.07 \\
22.91 \\
24.12 \\
23.10\end{array}$ & $\begin{array}{l}133.87 \\
140.73 \\
143.70 \\
144.90 \\
140.30\end{array}$ & $\begin{array}{l}119.46 \\
120.66 \\
120.79 \\
120.78 \\
117.20\end{array}$ \\
\hline $\begin{array}{l}199-D 2-6 \\
199-D 5-13 \\
199-D 8-5 \\
199-D 8-6 \\
199-F 1-2\end{array}$ & $\begin{array}{r}26.20 \\
27.18 \\
21.65 \\
28.84 \\
8.68\end{array}$ & $\begin{array}{l}143.04 \\
143.71 \\
137.92 \\
145.24 \\
121.30\end{array}$ & $\begin{array}{l}116.84 \\
116.53 \\
116.27 \\
116.40 \\
112.61\end{array}$ \\
\hline $\begin{array}{l}199-F 5-1 \\
199-F 7-1 \\
199-F 8-1 \\
199-F 8-4 \\
199-H 4-16\end{array}$ & $\begin{array}{r}11.34 \\
5.40 \\
10.85 \\
13.48 \\
15.37\end{array}$ & $\begin{array}{l}123.54 \\
118.32 \\
123.70 \\
125.25 \\
129.31\end{array}$ & $\begin{array}{l}112.21 \\
112.91 \\
112.85 \\
111.78 \\
113.93\end{array}$ \\
\hline $\begin{array}{l}199-H 5-1 A \\
199-H 6-1 \\
199-K-19 \\
199-K-20 \\
199-K-27\end{array}$ & $\begin{array}{l}13.59 \\
13.60 \\
10.84 \\
11.00 \\
22.91\end{array}$ & $\begin{array}{l}128.06 \\
127.44 \\
128.66 \\
128.61 \\
142.24\end{array}$ & $\begin{array}{l}114.47 \\
113.84 \\
117.82 \\
117.61 \\
119.34\end{array}$ \\
\hline $\begin{array}{l}199-K-34 \\
199-K-36 \\
199-K-37 \\
199-N-14 \\
199-N-26\end{array}$ & $\begin{array}{l}23.85 \\
30.07 \\
16.97 \\
21.18 \\
21.87\end{array}$ & $\begin{array}{l}142.67 \\
150.59 \\
134.66 \\
138.28 \\
139.06\end{array}$ & $\begin{array}{l}118.82 \\
120.52 \\
117.70 \\
117.09 \\
117.19\end{array}$ \\
\hline $\begin{array}{l}199-N-73 \\
199-N-74 \\
199-N-8 S \\
299-E 13-10 \\
299-E 13-14\end{array}$ & $\begin{array}{r}23.37 \\
21.01 \\
6.45 \\
102.47 \\
104.62\end{array}$ & $\begin{array}{l}141.19 \\
139.48 \\
123.47 \\
224.85 \\
224.85\end{array}$ & $\begin{array}{l}117.82 \\
118.47 \\
117.02 \\
122.38 \\
120.23\end{array}$ \\
\hline $\begin{array}{l}299-E 17-1 \\
299-E 17-10 \\
299-E 17-12 \\
299-E 17-13 \\
299-E 17-14\end{array}$ & $\begin{array}{l}96.97 \\
95.53 \\
97.67 \\
96.93 \\
97.78\end{array}$ & $\begin{array}{l}219.20 \\
217.85 \\
219.97 \\
219.23 \\
220.12\end{array}$ & $\begin{array}{l}122.24 \\
122.32 \\
122.30 \\
122.29 \\
122.34\end{array}$ \\
\hline
\end{tabular}




\section{WHC-EP-0394-10}

Appendix A: December 1994 Water Leve7 Measurement Data (Sheet 2 of 13)

\begin{tabular}{|c|c|c|c|}
\hline We 11 & $\begin{array}{l}\text { Depth to } \\
\text { Water (m) }\end{array}$ & $\begin{array}{l}\text { Elevation, } \\
\text { Adjusted Casing }\end{array}$ & $\begin{array}{l}\text { above ms } 1 \\
\quad \text { Water Leve1 }\end{array}$ \\
\hline $\begin{array}{l}299-E 17-15 \\
299-E 17-16 \\
299-E 17-17 \\
299-E 17-18 \\
299-E 17-5\end{array}$ & $\begin{array}{l}97.91 \\
97.37 \\
97.12 \\
97.34 \\
96.88\end{array}$ & $\begin{array}{l}220.00 \\
219.63 \\
219.43 \\
219.65 \\
219.06\end{array}$ & $\begin{array}{l}122.08 \\
122.26 \\
122.31 \\
122.31 \\
122.17\end{array}$ \\
\hline $\begin{array}{l}299-E 17-9 \\
299-E 18-1 \\
299-E 18-2 \\
299-E 18-3 \\
299-E 18-4\end{array}$ & $\begin{array}{l}96.48 \\
97.13 \\
97.52 \\
97.70 \\
97.55\end{array}$ & $\begin{array}{l}218.74 \\
219.53 \\
219.82 \\
220.08 \\
219.93\end{array}$ & $\begin{array}{l}122.26 \\
122.40 \\
122.31 \\
122.38 \\
122.39\end{array}$ \\
\hline $\begin{array}{l}299-E 23-1 \\
299-E 24-16 \\
299-E 24-18 \\
299-E 24-20 \\
299-E 24-7\end{array}$ & $\begin{array}{l}94.93 \\
96.59 \\
96.91 \\
87.62 \\
95.87\end{array}$ & $\begin{array}{l}217.35 \\
218.93 \\
219.24 \\
210.09 \\
218.24\end{array}$ & $\begin{array}{l}122.42 \\
122.34 \\
122.33 \\
122.47 \\
122.37\end{array}$ \\
\hline $\begin{array}{l}299-E 25-2 \\
299-E 25-32 P \\
299-E 25-34 \\
299-E 25-35 \\
299-E 25-36\end{array}$ & $\begin{array}{l}83.42 \\
81.77 \\
79.48 \\
83.13 \\
93.28\end{array}$ & $\begin{array}{l}205.94 \\
204.23 \\
202.04 \\
205.55 \\
215.61\end{array}$ & $\begin{array}{l}122.52 \\
122.46 \\
122.57 \\
122.42 \\
122.33\end{array}$ \\
\hline $\begin{array}{l}299-E 25-40 \\
299-E 25-41 \\
299-E 25-42 \\
299-E 25-46 \\
299-E 26-1\end{array}$ & $\begin{array}{l}80.49 \\
82.15 \\
85.82 \\
89.28 \\
65.55\end{array}$ & $\begin{array}{l}202.97 \\
204.66 \\
208.27 \\
211.78 \\
188.14\end{array}$ & $\begin{array}{l}122.48 \\
122.51 \\
122.45 \\
122.50 \\
122.59\end{array}$ \\
\hline $\begin{array}{l}299-E 26-10 \\
299-E 26-12 \\
299-E 26-13 \\
299-E 26-2 \\
299-E 26-9\end{array}$ & $\begin{array}{l}60.81 \\
69.67 \\
61.78 \\
71.21 \\
61.24\end{array}$ & $\begin{array}{l}183.33 \\
192.25 \\
184.41 \\
193.64 \\
183.76\end{array}$ & $\begin{array}{l}122.52 \\
122.58 \\
122.63 \\
122.43 \\
122.52\end{array}$ \\
\hline $\begin{array}{l}299-E 27-11 \\
299-E 27-12 \\
299-E 27-13 \\
299-E 27-14 \\
299-E 27-15\end{array}$ & $\begin{array}{l}73.73 \\
79.08 \\
81.49 \\
78.26 \\
76.65\end{array}$ & $\begin{array}{l}196.07 \\
201.52 \\
203.97 \\
200.72 \\
198.99\end{array}$ & $\begin{array}{l}122.35 \\
122.44 \\
122.48 \\
122.46 \\
122.34\end{array}$ \\
\hline
\end{tabular}




$$
\text { WHC-EP-0394-10 }
$$

Appendix A: December 1994 Water Level Measurement Data (Sheet 3 of 13)

\begin{tabular}{|c|c|c|c|}
\hline Well & $\begin{array}{l}\text { Depth to } \\
\text { Water (m) }\end{array}$ & $\begin{array}{r}\text { Elevation, } \\
\text { Adjusted Casing }\end{array}$ & $\begin{array}{l}\text { above } \mathrm{ms} 1 \\
\text { Water Level }\end{array}$ \\
\hline $\begin{array}{l}299-E 27-16 \\
299-E 27-17 \\
299-E 27-8 \\
299-E 27-9 \\
299-E 28-17\end{array}$ & $\begin{array}{l}76.49 \\
71.00 \\
72.02 \\
69.34 \\
93.57\end{array}$ & $\begin{array}{l}198.77 \\
193.46 \\
194.41 \\
191.78 \\
215.97\end{array}$ & $\begin{array}{l}122.28 \\
122.46 \\
122.39 \\
122.44 \\
122.40\end{array}$ \\
\hline $\begin{array}{l}299-E 28-26 \\
299-E 28-27 \\
299-E 28-28 \\
299-E 28-4 \\
299-E 28-6\end{array}$ & $\begin{array}{l}87.11 \\
85.02 \\
86.94 \\
88.30 \\
90.95\end{array}$ & $\begin{array}{l}209.48 \\
207.44 \\
209.26 \\
210.78 \\
213.39\end{array}$ & $\begin{array}{l}122.37 \\
122.42 \\
122.32 \\
122.49 \\
122.45\end{array}$ \\
\hline $\begin{array}{l}299-E 28-9 \\
299-E 32-1 \\
299-E 32-10 \\
299-E 32-2 \\
299-E 32-3\end{array}$ & $\begin{array}{l}91.17 \\
77.61 \\
72.04 \\
81.94 \\
83.89\end{array}$ & $\begin{array}{l}213.59 \\
200.00 \\
194.43 \\
204.23 \\
206.20\end{array}$ & $\begin{array}{l}122.43 \\
122.39 \\
122.38 \\
122.30 \\
122.31\end{array}$ \\
\hline $\begin{array}{l}299-E 32-4 \\
299-E 32-5 \\
299-E 32-6 \\
299-E 32-7 \\
299-E 32-8\end{array}$ & $\begin{array}{l}86.72 \\
85.50 \\
81.05 \\
78.31 \\
74.42\end{array}$ & $\begin{array}{l}209.06 \\
207.92 \\
203.44 \\
200.67 \\
196.76\end{array}$ & $\begin{array}{l}122.33 \\
122.42 \\
122.39 \\
122.37 \\
122.34\end{array}$ \\
\hline $\begin{array}{l}299-E 32-9 \\
299-E 33-13 \\
299-E 33-14 \\
299-E 33-15 \\
299-E 33-17\end{array}$ & $\begin{array}{l}73.56 \\
69.33 \\
67.17 \\
68.75 \\
70.13\end{array}$ & $\begin{array}{l}196.09 \\
191.52 \\
189.60 \\
191.20 \\
192.53\end{array}$ & $\begin{array}{l}122.53 \\
122.19 \\
122.44 \\
122.44 \\
122.40\end{array}$ \\
\hline $\begin{array}{l}299-E 33-18 \\
299-E 33-1 A \\
299-E 33-2 \\
299-E 33-21 \\
299-E 33-28\end{array}$ & $\begin{array}{l}76.23 \\
70.24 \\
70.10 \\
81.27 \\
80.12\end{array}$ & $\begin{array}{l}198.69 \\
192.67 \\
192.55 \\
203.73 \\
202.46\end{array}$ & $\begin{array}{l}122.46 \\
122.43 \\
122.45 \\
122.46 \\
122.34\end{array}$ \\
\hline $\begin{array}{l}299-E 33-29 \\
299-E 33-3 \\
299-E 33-30 \\
299-E 33-31 \\
299-E 33-32\end{array}$ & $\begin{array}{l}83.02 \\
69.78 \\
79.95 \\
74.93 \\
78.78\end{array}$ & $\begin{array}{l}205.37 \\
192.20 \\
202.30 \\
197.35 \\
201.17\end{array}$ & $\begin{array}{l}122.34 \\
122.42 \\
122.34 \\
122.43 \\
122.39\end{array}$ \\
\hline
\end{tabular}


WHC-EP-0394-10

Appendix A: December 1994 Water Level Measurement Data (Sheet 4 of 13)

\begin{tabular}{|c|c|c|c|}
\hline Well & $\begin{array}{l}\text { Depth to } \\
\text { Water (m) }\end{array}$ & $\begin{array}{l}\text { Elevation, } \\
\text { Adjusted Casing }\end{array}$ & $\begin{array}{l}\text { e msl } \\
\text { Water Level }\end{array}$ \\
\hline $\begin{array}{l}299-E 33-33 \\
299-E 33-34 \\
299-E 33-35 \\
299-E 33-36 \\
299-E 33-37\end{array}$ & $\begin{array}{l}72.77 \\
70.55 \\
73.66 \\
75.21 \\
76.72\end{array}$ & $\begin{array}{l}195.19 \\
193.11 \\
195.99 \\
197.11 \\
199.04\end{array}$ & $\begin{array}{l}122.42 \\
122.57 \\
122.33 \\
121.89 \\
122.32\end{array}$ \\
\hline $\begin{array}{l}299-E 33-38 \\
299-E 33-39 \\
299-E 33-4 \\
299-E 33-41 \\
299-E 33-42\end{array}$ & $\begin{array}{l}70.25 \\
67.68 \\
69.54 \\
77.31 \\
77.00\end{array}$ & $\begin{array}{l}192.62 \\
189.98 \\
191.38 \\
199.63 \\
199.43\end{array}$ & $\begin{array}{l}122.37 \\
122.30 \\
121.84 \\
122.32 \\
122.43\end{array}$ \\
\hline $\begin{array}{l}299-E 33-5 \\
299-E 33-7 \\
299-E 33-8 \\
299-E 34-1 \\
299-E 34-10\end{array}$ & $\begin{array}{l}71.02 \\
68.94 \\
75.97 \\
69.40 \\
72.55\end{array}$ & $\begin{array}{l}193.46 \\
191.34 \\
198.42 \\
191.86 \\
195.00\end{array}$ & $\begin{array}{l}122.45 \\
122.41 \\
122.46 \\
122.46 \\
122.46\end{array}$ \\
\hline $\begin{array}{l}299-E 34-11 \\
299-E 34-12 \\
299-E 34-2 \\
299-E 34-3 \\
299-E 34-5\end{array}$ & $\begin{array}{l}65.90 \\
72.31 \\
69.88 \\
63.95 \\
57.34\end{array}$ & $\begin{array}{l}188.35 \\
194.72 \\
192.27 \\
186.39 \\
180.07\end{array}$ & $\begin{array}{l}122.45 \\
122.40 \\
122.39 \\
122.44 \\
122.73\end{array}$ \\
\hline $\begin{array}{l}299-E 34-9 \\
299-E 35-2 \\
299-W 10-15 \\
299-W 10-16 \\
299-W 10-17\end{array}$ & $\begin{array}{l}69.18 \\
60.99 \\
65.72 \\
64.72 \\
64.04\end{array}$ & $\begin{array}{l}191.62 \\
183.60 \\
206.01 \\
205.06 \\
204.47\end{array}$ & $\begin{array}{l}122.44 \\
122.61 \\
140.30 \\
140.33 \\
140.44\end{array}$ \\
\hline $\begin{array}{l}299-W 10-18 \\
299-W 10-19 \\
299-W 10-2 \\
299-W 10-5 \\
299-W 10-8\end{array}$ & $\begin{array}{l}63.81 \\
68.20 \\
65.04 \\
64.36 \\
67.14\end{array}$ & $\begin{array}{l}204.50 \\
208.18 \\
205.43 \\
204.92 \\
207.36\end{array}$ & $\begin{array}{l}140.69 \\
139.98 \\
140.39 \\
140.56 \\
140.22\end{array}$ \\
\hline $\begin{array}{l}299-W 10-9 \\
299-W 11-10 \\
299-W 11-12 \\
299-W 11-23 \\
299-W 11-24\end{array}$ & $\begin{array}{l}65.37 \\
84.23 \\
66.73 \\
69.63 \\
69.28\end{array}$ & $\begin{array}{l}205.72 \\
222.17 \\
207.04 \\
209.75 \\
209.53\end{array}$ & $\begin{array}{l}140.35 \\
137.94 \\
140.31 \\
140.11 \\
140.25\end{array}$ \\
\hline
\end{tabular}


WHC-EP-0394-10

Appendix A: December 1994 Water Level Measurement Data (Sheet 5 of 13)

\begin{tabular}{|c|c|c|c|}
\hline Well & $\begin{array}{l}\text { Depth to } \\
\text { Water (m) }\end{array}$ & $\begin{array}{r}\text { Elevation, } \\
\text { Adjusted Casing }\end{array}$ & $\begin{array}{l}\text { above ms } 1 \\
\quad \text { Water Level }\end{array}$ \\
\hline $\begin{array}{l}299-W 11-27 \\
299-W 11-31 \\
299-W 11-6 \\
299-W 11-7 \\
299-W 11-9\end{array}$ & $\begin{array}{l}68.69 \\
76.37 \\
79.02 \\
76.12 \\
81.69\end{array}$ & $\begin{array}{l}208.87 \\
215.45 \\
218.31 \\
216.14 \\
220.35\end{array}$ & $\begin{array}{l}140.18 \\
139.08 \\
139.29 \\
140.02 \\
138.66\end{array}$ \\
\hline $\begin{array}{l}299-W 12-1 \\
299-W 14-1 \\
299-W 14-12 \\
299-W 14-5 \\
299-W 15-12\end{array}$ & $\begin{array}{l}84.81 \\
62.95 \\
63.45 \\
61.49 \\
63.54\end{array}$ & $\begin{array}{l}221.43 \\
203.85 \\
204.37 \\
203.18 \\
204.24\end{array}$ & $\begin{array}{l}136.62 \\
140.90 \\
140.93 \\
141.69 \\
140.70\end{array}$ \\
\hline $\begin{array}{l}299-W 15-13 \\
299-W 15-16 \\
299-W 15-18 \\
299-W 15-19 \\
299-W 15-2\end{array}$ & $\begin{array}{l}63.51 \\
67.86 \\
68.14 \\
69.89 \\
70.84\end{array}$ & $\begin{array}{l}204.25 \\
208.75 \\
209.00 \\
210.80 \\
210.53\end{array}$ & $\begin{array}{l}140.74 \\
140.90 \\
140.86 \\
140.91 \\
139.68\end{array}$ \\
\hline $\begin{array}{l}299-W 15-20 \\
299-W 15-22 \\
299-W 15-23 \\
299-W 15-24 \\
299-W 15-4\end{array}$ & $\begin{array}{l}72.11 \\
63.26 \\
72.46 \\
72.44 \\
60.73\end{array}$ & $\begin{array}{l}212.86 \\
204.45 \\
213.20 \\
213.17 \\
201.78\end{array}$ & $\begin{array}{l}140.75 \\
141.19 \\
140.74 \\
140.73 \\
141.05\end{array}$ \\
\hline $\begin{array}{l}299-W 15-5 \\
299-W 18-21 \\
299-W 18-23 \\
299-W 18-25 \\
299-W 18-26\end{array}$ & $\begin{array}{l}63.24 \\
63.06 \\
71.64 \\
62.26 \\
72.32\end{array}$ & $\begin{array}{l}204.42 \\
203.80 \\
212.39 \\
203.01 \\
213.07\end{array}$ & $\begin{array}{l}141.18 \\
140.73 \\
140.74 \\
140.74 \\
140.75\end{array}$ \\
\hline $\begin{array}{l}299-W 18-28 \\
299-W 18-3 \\
299-W 18-30 \\
299-W 18-31 \\
299-W 18-32\end{array}$ & $\begin{array}{l}66.44 \\
70.43 \\
64.20 \\
61.66 \\
65.40\end{array}$ & $\begin{array}{l}207.26 \\
209.70 \\
205.08 \\
202.44 \\
206.24\end{array}$ & $\begin{array}{l}140.82 \\
139.28 \\
140.88 \\
140.78 \\
140.84\end{array}$ \\
\hline $\begin{array}{l}299-W 19-12 \\
299-W 19-14 \\
299-W 19-15 \\
299-W 19-20 \\
299-W 19-21\end{array}$ & $\begin{array}{l}64.32 \\
71.69 \\
71.45 \\
72.43 \\
66.17\end{array}$ & $\begin{array}{l}205.21 \\
211.29 \\
211.31 \\
210.63 \\
206.90\end{array}$ & $\begin{array}{l}140.88 \\
139.60 \\
139.86 \\
138.20 \\
140.72\end{array}$ \\
\hline
\end{tabular}


WHC-EP-0394-10

Appendix A: December 1994 Water Level Measurement Data

(Sheet 6 of 13)

\begin{tabular}{|c|c|c|c|}
\hline We11 & $\begin{array}{l}\text { Depth to } \\
\text { Water (m) }\end{array}$ & $\begin{array}{l}\text { Elevation, } \\
\text { Adjusted Casing }\end{array}$ & $\begin{array}{l}\text { ve ms } 1 \\
\text { Water Level }\end{array}$ \\
\hline $\begin{array}{l}299-W 19-27 \\
299-W 19-28 \\
299-W 19-31 \\
299-W 19-32 \\
299-W 21-1\end{array}$ & $\begin{array}{l}67.10 \\
75.19 \\
64.60 \\
64.83 \\
75.57\end{array}$ & $\begin{array}{l}208.46 \\
214.30 \\
205.49 \\
205.71 \\
213.13\end{array}$ & $\begin{array}{l}141.36 \\
139.12 \\
140.89 \\
140.88 \\
137.56\end{array}$ \\
\hline $\begin{array}{l}299-W 22-39 \\
299-W 22-40 \\
299-W 22-41 \\
299-W 22-42 \\
299-W 22-43\end{array}$ & $\begin{array}{l}64.55 \\
72.55 \\
72.41 \\
72.34 \\
72.06\end{array}$ & $\begin{array}{l}203.69 \\
210.99 \\
210.84 \\
210.74 \\
210.72\end{array}$ & $\begin{array}{l}139.13 \\
138.44 \\
138.43 \\
138.41 \\
138.67\end{array}$ \\
\hline $\begin{array}{l}299-W 22-44 \\
299-W 22-45 \\
299-W 22-46 \\
299-W 23-11 \\
299-W 23-13\end{array}$ & $\begin{array}{l}67.01 \\
63.77 \\
65.51 \\
62.55 \\
63.25\end{array}$ & $\begin{array}{l}206.69 \\
203.06 \\
204.58 \\
202.43 \\
203.10\end{array}$ & $\begin{array}{l}139.68 \\
139.29 \\
139.07 \\
139.88 \\
139.85\end{array}$ \\
\hline $\begin{array}{l}299-W 23-14 \\
299-W 23-15 \\
299-W 26-10 \\
299-W 26-12 \\
299-W 26-7\end{array}$ & $\begin{array}{l}62.69 \\
60.44 \\
65.76 \\
67.12 \\
59.42\end{array}$ & $\begin{array}{l}202.39 \\
199.78 \\
204.48 \\
205.95 \\
198.73\end{array}$ & $\begin{array}{l}139.70 \\
139.34 \\
138.72 \\
138.83 \\
139.31\end{array}$ \\
\hline $\begin{array}{l}299-W 26-8 \\
299-W 26-9 \\
299-W 6-1 \\
299-W 6-10 \\
299-W 6-11\end{array}$ & $\begin{array}{l}64.17 \\
60.53 \\
75.53 \\
78.92 \\
75.93\end{array}$ & $\begin{array}{l}203.09 \\
199.39 \\
214.13 \\
217.16 \\
214.23\end{array}$ & $\begin{array}{l}138.92 \\
138.86 \\
138.60 \\
138.25 \\
138.30\end{array}$ \\
\hline $\begin{array}{l}299-W 6-12 \\
299-W 6-2 \\
299-W 6-4 \\
299-W 6-7 \\
299-W 7-1\end{array}$ & $\begin{array}{l}72.45 \\
71.94 \\
74.72 \\
78.45 \\
71.17\end{array}$ & $\begin{array}{l}211.08 \\
211.06 \\
213.74 \\
216.49 \\
210.53\end{array}$ & $\begin{array}{l}138.62 \\
139.12 \\
139.02 \\
138.05 \\
139.36\end{array}$ \\
\hline $\begin{array}{l}299-W 7-10 \\
299-W 7-11 \\
299-W 7-12 \\
299-W 7-2 \\
299-W 7-4\end{array}$ & $\begin{array}{l}71.28 \\
68.44 \\
70.33 \\
66.89 \\
65.19\end{array}$ & $\begin{array}{l}210.21 \\
207.71 \\
209.68 \\
205.92 \\
204.80\end{array}$ & $\begin{array}{l}138.93 \\
139.27 \\
139.35 \\
139.03 \\
139.61\end{array}$ \\
\hline
\end{tabular}


WHC-EP-0394-10

Appendix A: December 1994 Water Level Measurement Data (Sheet 7 of 13)

\begin{tabular}{|c|c|c|c|}
\hline Well & $\begin{array}{l}\text { Depth to } \\
\text { Water }(\mathrm{m})\end{array}$ & $\begin{array}{l}\text { Elevation, } \\
\text { Adjusted Casing }\end{array}$ & $\begin{array}{l}\text { e ms1 } \\
\text { Water Leve1 }\end{array}$ \\
\hline $\begin{array}{l}299-W 7-5 \\
299-W 7-6 \\
299-W 7-7 \\
299-W 7-9 \\
299-W 8-1\end{array}$ & $\begin{array}{l}66.27 \\
68.01 \\
66.83 \\
71.44 \\
74.26\end{array}$ & $\begin{array}{l}205.15 \\
206.85 \\
205.72 \\
210.95 \\
213.77\end{array}$ & $\begin{array}{l}138.88 \\
138.84 \\
138.89 \\
139.51 \\
139.51\end{array}$ \\
\hline $\begin{array}{l}3099-47-18 \mathrm{~B} \\
399-1-1 \\
399-1-10 \mathrm{~A} \\
399-1-11 \\
399-1-12\end{array}$ & $\begin{array}{r}10.36 \\
10.71 \\
9.80 \\
10.82 \\
12.95\end{array}$ & $\begin{array}{l}114.36 \\
114.81 \\
113.86 \\
115.13 \\
117.17\end{array}$ & $\begin{array}{l}104.00 \\
104.09 \\
104.06 \\
104.31 \\
104.22\end{array}$ \\
\hline $\begin{array}{l}399-1-14 A \\
399-1-15 \\
399-1-16 A \\
399-1-17 A \\
399-1-18 A\end{array}$ & $\begin{array}{l}12.46 \\
11.28 \\
12.21 \\
10.88 \\
14.62\end{array}$ & $\begin{array}{l}116.81 \\
115.68 \\
116.27 \\
115.04 \\
119.11\end{array}$ & $\begin{array}{l}104.35 \\
104.40 \\
104.06 \\
104.17 \\
104.49\end{array}$ \\
\hline $\begin{array}{l}399-1-2 \\
399-1-3 \\
399-1-4 \\
399-1-5 \\
399-1-6\end{array}$ & $\begin{array}{r}13.78 \\
13.16 \\
11.70 \\
11.54 \\
9.65\end{array}$ & $\begin{array}{l}117.20 \\
117.26 \\
116.00 \\
115.75 \\
113.94\end{array}$ & $\begin{array}{l}103.42 \\
104.10 \\
104.31 \\
104.21 \\
104.29\end{array}$ \\
\hline $\begin{array}{l}399-1-7 \\
399-1-8 \\
399-2-2 \\
399-2-3 \\
399-3-1\end{array}$ & $\begin{array}{l}13.47 \\
13.19 \\
11.03 \\
10.40 \\
13.13\end{array}$ & $\begin{array}{l}117.53 \\
117.31 \\
115.07 \\
114.43 \\
117.16\end{array}$ & $\begin{array}{l}104.06 \\
104.12 \\
104.04 \\
104.03 \\
104.03\end{array}$ \\
\hline $\begin{array}{l}399-3-10 \\
399-3-6 \\
399-3-9 \\
399-4-10 \\
399-4-11\end{array}$ & $\begin{array}{l}13.46 \\
15.60 \\
14.26 \\
11.40 \\
19.25\end{array}$ & $\begin{array}{l}117.45 \\
119.73 \\
118.29 \\
115.38 \\
123.28\end{array}$ & $\begin{array}{l}103.99 \\
104.13 \\
104.03 \\
103.98 \\
104.03\end{array}$ \\
\hline $\begin{array}{l}399-6-1 \\
399-8-1 \\
699-10-54 A \\
699-10-E 12 \\
699-101-48 B\end{array}$ & $\begin{array}{r}14.32 \\
16.40 \\
31.37 \\
22.56 \\
3.86\end{array}$ & $\begin{array}{l}118.50 \\
120.74 \\
157.40 \\
131.33 \\
118.90\end{array}$ & $\begin{array}{l}104.19 \\
104.34 \\
126.03 \\
108.77 \\
115.04\end{array}$ \\
\hline
\end{tabular}




\section{WHC-EP-0394-10}

Appendix A: December 1994 Water Level Measurement Data (Sheet 8 of 13)

\begin{tabular}{|c|c|c|c|}
\hline Well & $\begin{array}{l}\text { Depth to } \\
\text { Water }(\mathrm{m})\end{array}$ & $\begin{array}{r}\text { Elevation, } \\
\text { Adjusted Casing }\end{array}$ & $\begin{array}{l}\text { above ms } 1 \\
\text { Water Level }\end{array}$ \\
\hline $\begin{array}{l}699-11-45 A \\
699-14-38 \\
699-14-47 \\
699-15-15 A \\
699-15-26\end{array}$ & $\begin{array}{l}50.77 \\
33.65 \\
53.39 \\
45.70 \\
37.91\end{array}$ & $\begin{array}{l}176.35 \\
156.94 \\
178.99 \\
166.77 \\
159.66\end{array}$ & $\begin{array}{l}125.58 \\
123.29 \\
125.59 \\
121.07 \\
121.75\end{array}$ \\
\hline $\begin{array}{l}699-17-5 \\
699-17-70 \\
699-19-43 \\
699-19-58 \\
699-19-88\end{array}$ & $\begin{array}{l}14.03 \\
27.04 \\
45.45 \\
47.18 \\
39.81\end{array}$ & $\begin{array}{l}132.04 \\
171.66 \\
168.12 \\
174.63 \\
196.43\end{array}$ & $\begin{array}{l}118.01 \\
144.61 \\
122.67 \\
127.45 \\
156.62\end{array}$ \\
\hline $\begin{array}{l}699-2-3 \\
699-2-33 A \\
699-20-20 \\
699-20-E 12 \\
699-24-33\end{array}$ & $\begin{array}{l}26.69 \\
40.32 \\
32.35 \\
24.43 \\
37.56\end{array}$ & $\begin{array}{l}145.43 \\
163.49 \\
154.10 \\
133.27 \\
159.80\end{array}$ & $\begin{array}{l}118.74 \\
123.16 \\
121.76 \\
108.85 \\
122.23\end{array}$ \\
\hline $\begin{array}{l}699-24-34 A \\
699-24-34 B \\
699-24-34 C \\
699-24-35 \\
699-25-34 A\end{array}$ & $\begin{array}{l}40.50 \\
40.37 \\
40.10 \\
41.99 \\
39.59\end{array}$ & $\begin{array}{l}162.73 \\
162.61 \\
162.33 \\
164.23 \\
161.64\end{array}$ & $\begin{array}{l}122.23 \\
122.24 \\
122.23 \\
122.24 \\
122.05\end{array}$ \\
\hline $\begin{array}{l}699-25-34 B \\
699-25-34 C \\
699-25-34 D \\
699-25-55 \\
699-25-70\end{array}$ & $\begin{array}{l}39.59 \\
40.98 \\
41.71 \\
80.64 \\
56.22\end{array}$ & $\begin{array}{l}161.36 \\
163.21 \\
163.95 \\
206.24 \\
191.93\end{array}$ & $\begin{array}{l}121.77 \\
122.23 \\
122.24 \\
125.60 \\
135.71\end{array}$ \\
\hline $\begin{array}{l}699-26-15 A \\
699-26-33 \\
699-26-34 A \\
699-26-34 B \\
699-26-35 A\end{array}$ & $\begin{array}{l}13.96 \\
41.12 \\
38.93 \\
39.38 \\
40.24\end{array}$ & $\begin{array}{l}134.92 \\
163.27 \\
161.06 \\
161.63 \\
162.35\end{array}$ & $\begin{array}{l}120.96 \\
122.15 \\
122.13 \\
122.24 \\
122.11\end{array}$ \\
\hline $\begin{array}{l}699-26-89 \\
699-27-8 \\
699-28-52 A \\
699-29-78 \\
699-3-45\end{array}$ & $\begin{array}{l}55.69 \\
22.32 \\
85.80 \\
57.56 \\
28.15\end{array}$ & $\begin{array}{l}199.06 \\
141.94 \\
208.61 \\
197.21 \\
153.78\end{array}$ & $\begin{array}{l}143.37 \\
119.61 \\
122.81 \\
139.65 \\
125.64\end{array}$ \\
\hline
\end{tabular}


WHC-EP-0394-10

Appendix A: December 1994 Water Level Measurement Data (Sheet 9 of 13)

We11

$699-32-43$
$699-32-62$
$699-33-42$
$699-33-56$
$699-34-39 A$

$699-34-41 B$

$699-34-51$

$699-34-88$

$699-35-66$

699-35-70

$699-35-9$

$699-36-61 A$

$699-37-43$

$699-38-65$

$699-38-70$

699-40-1

699-40-33A

699-40-62

699-41-23

699-41-42

699-42-37

699-42-40B

699-42-41

699-43-104

699-43-40

699-43-42J

699-43-45

699-43-89

699-44-39B

699-44-42

699-44-43B

699-44-64

699-45-42

699-46-21B

699-47-35A
Depth to

Water (m)

35.09
85.22
34.88
95.83
41.32

51.62

102.00

50.65

88.01

74.95

35.32

103.88

87.74

99.06

80.00

23.30

32.91

104.73

21.37

70.98

31.81

38.15

44.83

83.40

37.07

50.09

59.49

54.97

28.27

48.70

50.66

97.62

49.31

40.24

19.42
Elevation, $m$ above $\mathrm{ms}$ Adjusted Casing Water Level

157.47

215.53

157.28

218.54

163.70

174.01

224.51

192.88

221.42

211.29

152.35

228.02

210.36

229.68

216.67

133.72

157.90

227.90

142.19

196.26

158.31

166.56

172.91

233.50

165.26

177.30

182.17

196.34

156.48

176.55

176.82

221.16

175.97

159.11

145.19
122.37

130.31

122.40

122.71

122.38

122.39

122.51

142.23

133.41

136.35

117.03

124.15

122.63

130.61

136.67

110.42

124.99

123.17

120.82

125.29

126.51

128.41

128.09

150.10

128.20

127.21

122.69

141.37

128.21

127.84

126.16

123.54

126.66

118.88

125.78 


\section{WHC-EP-0394-10}

Appendix A: December 1994 Water Level Measurement Data

(Sheet 10 of 13)

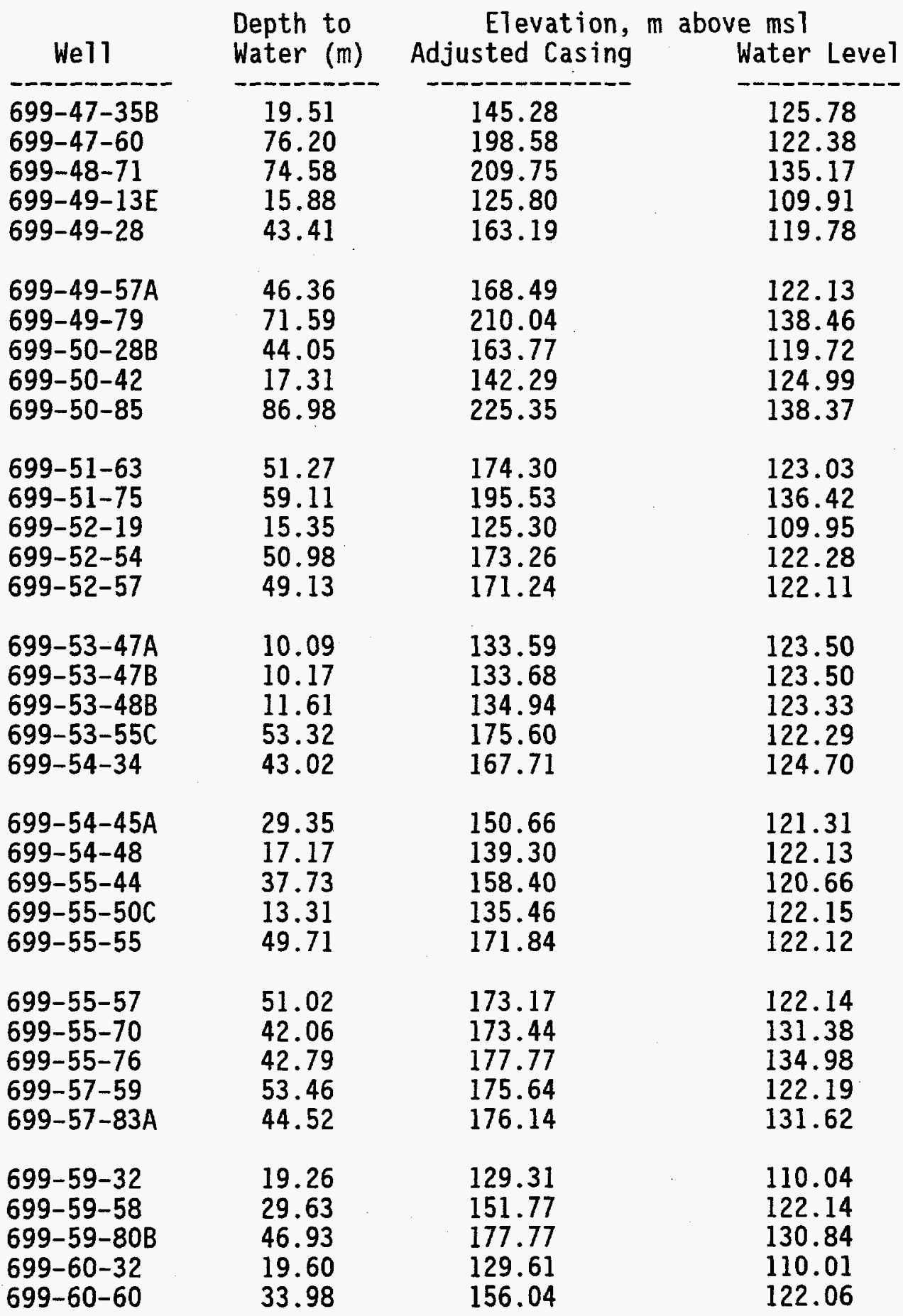


WHC-EP-0394-10

Appendix A: December 1994 Water Level Measurement Data (Sheet 11 of 13)

\begin{tabular}{|c|c|c|c|}
\hline We11 & $\begin{array}{l}\text { Depth to } \\
\text { Water }(\mathrm{m})\end{array}$ & $\begin{array}{r}\text { Elevation, } \\
\text { Adjusted Casing }\end{array}$ & $\begin{array}{l}\text { m above msl } \\
\text { Water Level }\end{array}$ \\
\hline $\begin{array}{l}699-61-62 \\
699-61-66 \\
699-62-31 \\
699-62-43 A \\
699-63-25 A\end{array}$ & $\begin{array}{l}29.57 \\
37.34 \\
22.31 \\
11.21 \\
10.57\end{array}$ & $\begin{array}{l}151.63 \\
159.16 \\
132.30 \\
131.78 \\
120.44\end{array}$ & $\begin{array}{l}122.06 \\
121.83 \\
110.00 \\
120.57 \\
109.87\end{array}$ \\
\hline $\begin{array}{l}699-63-51 \\
699-63-55 \\
699-63-58 \\
699-63-90 \\
699-64-62\end{array}$ & $\begin{array}{r}7.96 \\
8.39 \\
28.22 \\
34.67 \\
30.84\end{array}$ & $\begin{array}{l}129.38 \\
129.98 \\
149.89 \\
155.82 \\
152.44\end{array}$ & $\begin{array}{l}121.42 \\
121.59 \\
121.67 \\
121.15 \\
121.61\end{array}$ \\
\hline $\begin{array}{l}699-65-22 \\
699-65-50 \\
699-65-59 A \\
699-65-72 \\
699-65-83\end{array}$ & $\begin{array}{r}9.71 \\
20.95 \\
32.90 \\
43.59 \\
27.19\end{array}$ & $\begin{array}{l}119.18 \\
142.33 \\
154.51 \\
164.64 \\
148.01\end{array}$ & $\begin{array}{l}109.47 \\
121.38 \\
121.61 \\
121.05 \\
120.82\end{array}$ \\
\hline $\begin{array}{l}699-66-103 \\
699-66-38 \\
699-66-58 \\
699-66-64 \\
699-67-51\end{array}$ & $\begin{array}{l}20.68 \\
10.39 \\
31.82 \\
32.72 \\
38.49\end{array}$ & $\begin{array}{l}141.31 \\
132.94 \\
153.37 \\
154.16 \\
159.85\end{array}$ & $\begin{array}{l}120.63 \\
122.55 \\
121.55 \\
121.44 \\
121.37\end{array}$ \\
\hline $\begin{array}{l}699-67-86 \\
699-67-98 \\
699-68-105 \\
699-69-38 \\
699-70-23\end{array}$ & $\begin{array}{r}23.27 \\
18.21 \\
17.82 \\
6.37 \\
9.38\end{array}$ & $\begin{array}{l}143.98 \\
138.81 \\
138.42 \\
128.91 \\
119.35\end{array}$ & $\begin{array}{l}120.72 \\
120.60 \\
120.60 \\
122.54 \\
109.97\end{array}$ \\
\hline $\begin{array}{l}699-70-68 \\
699-71-30 \\
699-71-52 \\
699-72-73 \\
699-72-88\end{array}$ & $\begin{array}{r}39.23 \\
9.35 \\
38.23 \\
26.63 \\
11.94\end{array}$ & $\begin{array}{l}160.35 \\
122.10 \\
159.40 \\
147.09 \\
132.07\end{array}$ & $\begin{array}{l}121.12 \\
112.75 \\
121.17 \\
120.47 \\
120.12\end{array}$ \\
\hline $\begin{array}{l}699-72-92 \\
699-73-61 \\
699-74-44 \\
699-74-48 \\
699-77-36\end{array}$ & $\begin{array}{l}16.78 \\
40.83 \\
14.96 \\
27.66 \\
11.20\end{array}$ & $\begin{array}{l}137.02 \\
161.98 \\
135.67 \\
148.39 \\
125.64\end{array}$ & $\begin{array}{l}120.24 \\
121.15 \\
120.71 \\
120.73 \\
114.44\end{array}$ \\
\hline
\end{tabular}


WHC-EP-0394-10

Appendix A: December 1994 Water Leve1 Measurement Data (Sheet 12 of 13)

\begin{tabular}{|c|c|c|c|}
\hline Well & $\begin{array}{l}\text { Depth to } \\
\text { Water (m) }\end{array}$ & $\begin{array}{r}\text { Elevation, } \\
\text { Adjusted Casing }\end{array}$ & $\begin{array}{l}\text { ve msl } \\
\text { Water Leve1 }\end{array}$ \\
\hline $\begin{array}{l}699-77-54 \\
699-78-62 \\
699-8-17 \\
699-8-25 \\
699-8-32\end{array}$ & $\begin{array}{l}25.88 \\
23.26 \\
38.21 \\
33.83 \\
47.38\end{array}$ & $\begin{array}{l}146.32 \\
143.16 \\
159.24 \\
155.23 \\
168.98\end{array}$ & $\begin{array}{l}120.43 \\
119.91 \\
121.03 \\
121.40 \\
121.60\end{array}$ \\
\hline $\begin{array}{l}699-80-435 \\
699-81-38 \\
699-81-58 \\
699-83-36 \\
699-83-47\end{array}$ & $\begin{array}{r}7.81 \\
8.45 \\
14.45 \\
12.60 \\
14.41\end{array}$ & $\begin{array}{l}125.81 \\
123.86 \\
133.96 \\
127.56 \\
132.67\end{array}$ & $\begin{array}{l}117.99 \\
115.41 \\
119.51 \\
114.96 \\
118.26\end{array}$ \\
\hline $\begin{array}{l}699-86-42 \\
699-87-42 A \\
699-87-55 \\
699-88-41 \\
699-89-35\end{array}$ & $\begin{array}{r}7.86 \\
10.08 \\
22.39 \\
10.43 \\
8.30\end{array}$ & $\begin{array}{l}124.91 \\
126.94 \\
140.09 \\
126.79 \\
121.32\end{array}$ & $\begin{array}{l}117.06 \\
116.86 \\
117.70 \\
116.36 \\
113.02\end{array}$ \\
\hline $\begin{array}{l}699-9-E 2 \\
699-90-45 \\
699-96-49 \\
699-97-43 \\
699-97-51 A\end{array}$ & $\begin{array}{r}14.00 \\
11.57 \\
11.68 \\
13.29 \\
6.64\end{array}$ & $\begin{array}{l}127.43 \\
128.48 \\
127.78 \\
128.58 \\
122.61\end{array}$ & $\begin{array}{l}113.44 \\
116.91 \\
116.10 \\
115.28 \\
115.97\end{array}$ \\
\hline $\begin{array}{l}699-S 12-29 \\
699-S 12-3 \\
699-S 14-20 A \\
699-S 19-11 \\
699-S 19-E 13\end{array}$ & $\begin{array}{l}25.55 \\
16.66 \\
28.09 \\
28.56 \\
15.58\end{array}$ & $\begin{array}{l}148.64 \\
132.75 \\
150.19 \\
147.44 \\
120.25\end{array}$ & $\begin{array}{l}123.10 \\
116.09 \\
122.10 \\
118.88 \\
104.67\end{array}$ \\
\hline $\begin{array}{l}699-S 19-E 14 \\
699-S 27-E 14 \\
699-S 28-E 12 \\
699-S 3-25 \\
699-S 3-E 12\end{array}$ & $\begin{array}{r}9.40 \\
17.86 \\
13.79 \\
38.16 \\
13.40\end{array}$ & $\begin{array}{l}113.95 \\
121.82 \\
118.80 \\
159.56 \\
121.28\end{array}$ & $\begin{array}{l}104.55 \\
103.96 \\
105.01 \\
121.40 \\
107.88\end{array}$ \\
\hline $\begin{array}{l}699-S 30-E 10 \\
699-S 30-E 10 \\
699-S 30-E 15 \\
699-S 31-1 \\
699-S 31-E 10\end{array}$ & $\begin{array}{l}12.87 \\
12.66 \\
18.05 \\
25.08 \\
10.65\end{array}$ & $\begin{array}{l}119.55 \\
119.48 \\
122.12 \\
140.20 \\
117.20\end{array}$ & $\begin{array}{l}106.68 \\
106.82 \\
104.07 \\
115.13 \\
106.55\end{array}$ \\
\hline
\end{tabular}


WHC-EP-0394-10

Appendix A: December 1994 Water Level Measurement Data (Sheet 13 of 13)

\begin{tabular}{|c|c|c|c|}
\hline Well & $\begin{array}{l}\text { Depth to } \\
\text { Water (m) }\end{array}$ & $\begin{array}{r}\text { Elevation, } \\
\text { Adjusted Casing }\end{array}$ & $\begin{array}{l}\text { Ie ms1 } \\
\text { Water Level }\end{array}$ \\
\hline $\begin{array}{l}699-S 31-E 10 \\
699-S 31-E 10 \\
699-S 31-E 10 \\
699-S 32-E 13 \\
699-S 32-E 13\end{array}$ & $\begin{array}{r}10.16 \\
9.95 \\
9.27 \\
13.44 \\
14.65\end{array}$ & $\begin{array}{l}116.94 \\
116.69 \\
115.98 \\
118.99 \\
120.29\end{array}$ & $\begin{array}{l}106.77 \\
106.74 \\
106.71 \\
105.55 \\
105.64\end{array}$ \\
\hline $\begin{array}{l}699-S 34-E 10 \\
699-S 34-E 15 \\
699-S 36-E 12 \\
699-S 36-E 13 \\
699-\$ 37-E 14\end{array}$ & $\begin{array}{r}8.53 \\
17.13 \\
14.09 \\
14.21 \\
16.98\end{array}$ & $\begin{array}{l}116.52 \\
123.31 \\
121.62 \\
121.69 \\
124.43\end{array}$ & $\begin{array}{l}107.98 \\
106.18 \\
107.53 \\
107.48 \\
107.45\end{array}$ \\
\hline $\begin{array}{l}699-S 38-E 11 \\
699-S 38-E 12 \\
699-S 38-E 12 \\
699-S 40-E 14 \\
699-S 41-E 12\end{array}$ & $\begin{array}{l}13.46 \\
15.73 \\
15.75 \\
14.72 \\
14.50\end{array}$ & $\begin{array}{l}121.48 \\
123.41 \\
123.43 \\
122.78 \\
122.50\end{array}$ & $\begin{array}{l}108.02 \\
107.68 \\
107.68 \\
108.06 \\
108.00\end{array}$ \\
\hline $\begin{array}{l}699-S 41-E 13 \\
699-S 41-E 13 \\
699-S 43-E 12 \\
699-S 6-E 4 D \\
699-S 7-34\end{array}$ & $\begin{array}{l}17.22 \\
17.11 \\
15.83 \\
17.78 \\
36.20\end{array}$ & $\begin{array}{l}125.12 \\
124.98 \\
123.61 \\
131.21 \\
160.67\end{array}$ & $\begin{array}{l}107.90 \\
107.87 \\
107.78 \\
113.43 \\
124.47\end{array}$ \\
\hline $699-58-19$ & 32.78 & 153.56 & 120.79 \\
\hline
\end{tabular}


WHC-EP-0394-10

This page intentionally left blank. 
WHC-EP-0394-10

\section{Distribution}

Number of Copies

\section{offsite}

$1 \quad$ U.S. Geological Survey

B. D. Drost

1201 Pacific Ave. Suite 600

Tacoma, Wa. 98402

\section{Onsite}

$3 \quad$ U.S. Department of Enerqy Richland, Operations Office

M. J. Furman R3-80

A. J. Lassila \$7-52

K. M. Thompson A5-19

2 Washington State Department

\begin{tabular}{ll}
\hline of Ecology & $N 1-08$ \\
\hline S. Leja & $N 1-08$
\end{tabular}

$2 \quad$ U.S. Environmental Protection Agency

D. R. Sherwood (2) B5-01

6 Pacific Northwest Laboratory
M. A. Chamness (2)
K6-84
S. M. Goodwin
K6-84
G. V. Last
K6-84
S. P. Luttre11
K6-96
D. R. Newcomer
K6-96

45 Westinghouse Hanford Company

M. R. Adams H6-30

D. J. Alexander H6-06

D. B. Barnett H6-06

J. A. Caggiano H6-06

L. B. Collard H6-0l

J. D. Davis H6-01

L. P. Diediker T1-30

J. J. Dorian H6-30

G. L. Dunford Rl-51

J. W. Fassett H6-06

M. G. Gardner N3-06

M. J. Hartman H6-06 
WHC-EP-0394-10

Westinghouse Hanford Company (cont)

F. N. Hodges

D. G. Horton

H6-06

V. G. Johnson

H6-06

J. W. Lindberg

H6-06

A. H. Lu

H6-06

R. B. Mercer

$\mathrm{HO}-36$

S. E. Myers

H6-06

. M. Narbutovskih H6-06

R. S. Pavlina T7-39

S. M. Price H6-23

S. P. Reidel H6-06

K. D. Reynolds H6-06

A. L. Schatz N3-05

J. S. Schmid H6-06

J. A. Serkowski (10) H6-06

M. D. Sweeney H6-06

R. R. Thompson H6-32

E. C. Thornton H6-06

J. M. Votava H6-06

$3 \quad \mathrm{CH} 2 \mathrm{M} \mathrm{Hill}$

J. V. Borghese H4-9O

W. J. McMahon H4-90

R. E. Peterson H6-05

5 Bechtel Hanford Incorporated

G. E. Fitzgibbon H6-07

K. R. Fecht (2) H4-85

B. H. Ford

S. J. Trent

H4-85

H4-85

$4 \quad$ IT

G. L. Kasza H6-04

L. C. Swanson (2) H6-03

D. C. Weekes H4-82

Central Files (2) A3-88

OSTI (2) A3-36

EPIC (2) H6-08

Public Reading Room A1-65 